Canadian University Music Review

Canadian University Music Review

Revue de musique des universités canadiennes

\title{
Méthodologie schenkérienne et apprentissage de l’analyse musicale
}

\section{Carmen Sabourin}

\section{Numéro 14, 1994}

URI : https://id.erudit.org/iderudit/1014313ar

DOI : https://doi.org/10.7202/1014313ar

Aller au sommaire du numéro

\section{Éditeur(s)}

Canadian University Music Society / Société de musique des universités canadiennes

\section{ISSN}

0710-0353 (imprimé)

2291-2436 (numérique)

Découvrir la revue

Citer cet article

Sabourin, C. (1994). Méthodologie schenkérienne et apprentissage de l'analyse musicale. Canadian University Music Review / Revue de musique des universités canadiennes, (14), 98-145. https://doi.org/10.7202/1014313ar

\section{Résumé de l'article}

Nous considérons ici les visées didactiques et certains aspects structurels de la théorie de Heinrich Schenker dans la perspective de l'apprentissage de l'analyse musicale en milieu universitaire de tradition musicologique française. Dans un premier temps, nous posons la question de la pertinence de l'enseignement de l'analyse schenkérienne. Nous mettons en évidence les aspects de la théorie qui la distinguent des théories tonales antérieures et qui représentent des acquis substantiels pour l'étude de la tonalité. Puis, nous évaluons les difficultés inhérentes à la diffusion des idées de Schenker dans les milieux pédagogiques, soit la complexité de son œuvre, l'absence d'un traité d'harmonie à teneur schenkérienne en langue française et l'exploration des niveaux de structure intermédiaire (Mittelgrund). Par la suite, nous définissons les étapes préalables à l'apprentissage des techniques schenkériennes, notamment l'étude des espèces fuxiennes et de l'harmonie, cette dernière enseignée dans une perspective linéaire. Enfin, nous analysons l'Invention 12 en la majeur, BWV 783, de Johann Sebastian Bach. L'analyse met en évidence la relation entre les principes contrapuntiques présentés dans les étapes préalables et les différents niveaux de structure de l'œuvre. Un graphe schenkérien illustre l'interdépendance des niveaux de structure.
All Rights Reserved @ Canadian University Music Society / Société de musique des universités canadiennes, 1994
Ce document est protégé par la loi sur le droit d'auteur. L’utilisation des services d'Érudit (y compris la reproduction) est assujettie à sa politique d'utilisation que vous pouvez consulter en ligne.

https://apropos.erudit.org/fr/usagers/politique-dutilisation/ 


\title{
MÉTHODOLOGIE SCHENKÉRIENNE ET APPRENTISSAGE DE L'ANALYSE MUSICALE
}

\author{
Carmen Sabourin
}

Les idées du théoricien autrichien Heinrich Schenker (1868-1935) sont apparues en Amérique du Nord au cours des années 30 à la faveur de l'émigration de ses étudiants aux États-Unis. Elles se sont peu à peu répandues, non sans difficultés, dans les universités américaines, puis dans certaines universités canadiennesanglaises, où elles représentent à ce jour l'approche théorique dominante en musique tonale ${ }^{1}$. Il en va tout autrement dans les institutions de langue française. En effet, on ne voit apparaitre que très lentement les signes d'intégration des techniques d'analyse schenkérienne dans les milieux d'enseignement francophones. L'absence de traduction française des ouvrages de Schenker ${ }^{2}$ et le manque flagrant de spécialistes francophones des techniques schenkériennes n'expliquent pourtant qu'en partie ce phénomène.

On peut facilement identifier certains des facteurs à l'origine de cette absence d'intérêt. L'un d'eux repose sur le fait que la théorie schenkérienne est le résultat d'une conception nouvelle du contrepoint et de l'harmonie. L'intégration des idées de Schenker en milieu universitaire exige donc une sérieuse remise en question de l'enseignement de ces disciplines. Il va sans dire que de tels bouleversements suscitent une inquiétude fort légitime que nous tenterons de dissiper.

Nous proposons dans cet article de poser la question de la pertinence des visées didactiques de la théorie schenkérienne. Il est évidemment impossible de présenter un exposé complet de la théorie, et tel n'est pas notre objectif ${ }^{3}$. Notre

1 Le lecteur trouvera un historique de la dissémination des idées de Schenker aux États-Unis et en Europe dans David Beach, « The Current State of Schenkerian Research », Acta musicologica 57 (1985) : 275-99. Voir aussi William Rothstein, « The Americanization of Heinrich Schenker », dans Schenker Studies, éd. par Hedi Siegel (Cambridge : Cambridge University Press, 1990), 193-203.

2 Le musicologue belge Nicolas Meeùs travaille présentement à une traduction française du dernier ouvrage de Schenker, Neue musikalische Theorien und Phantasien III : Der freie Satz (Vienne : Universal Edition, 1935, 1956) ; l'ouvrage existe déjà en traduction anglaise dans une édition de Ernst Oster sous le titre Free Composition (New York : Longman, 1979).

3 Pour une présentation des points fondamentaux de la théorie schenkérienne en français, voir Célestin Deliège, Les fondements de la musique tonale (Paris : J. C. Lattès, 1984), 51-82. Pour un exposé littéral de la théorie, voir Oswald Jonas, Introduction to the Theory of Heinrich 
propos, fondé sur notre propre expérience d'enseignement de l'analyse schenkérienne en milieu universitaire, s'adresse plutôt au lecteur non praticien mais désireux d'acquérir un ensemble de paramètres qui lui permettront d'évaluer le potentiel pédagogique de la théorie schenkérienne ${ }^{4}$. Nous tenterons donc, dans un premier temps, de répondre à la question de la pertinence de l'enseignement de l'analyse schenkérienne à l'université ; puis, nous évaluerons les difficultés inhérentes à la diffusion des idées de Schenker dans les milieux pédagogiques ; par la suite, nous définirons les étapes préalables à l'apprentissage des techniques schenkériennes; enfin, nous analyserons l'Inventio 12 en la majeur, BWV 783, de Johann Sebastian Bach à la lumière des observations dégagées antérieurement.

Au sujet de la terminologie de Schenker, nous avons choisi de suivre de très près les équivalents anglais et, lorsqu'un terme apparait pour la première fois, d'offrir la version allemande dans le texte. Les lecteurs qui ont fréquenté l'œuvre de Schenker en anglais et en allemand pourront ainsi s'orienter facilement. La recherche d'équivalents français à la terminologie schenkérienne pose problème. Elle ne peut constituer une entreprise individuelle et continuera de poser problème tant que ne s'uniront pas chercheurs, spécialistes et traducteurs dans un effort collectif. Donc, nous ne prétendons nullement que les termes français employés dans cet article constituent des choix définitifs ${ }^{5}$.

\section{Pertinence de l'enseignement de l'analyse schenkérienne}

La complexité de l'œuvre de Schenker et les années d'étude sérieuse et de pratique qu'elle impose au musicien qui s'y intéresse sont au cœur des résistances

Schenker, trad. et éd. par John Rothgeb (New York : Schirmer Books, 1982) ; l'édition originale est parue sous le titre de Das Wesen des musikalischen Kunstwerks : eine Einführung in die Lehre Heinrich Schenkers (Vienne : Saturn-Verlag, 1934).

4 Sur l'enseignement de la théorie schenkérienne à l'université, voir David Beach, « Schenker's Theories : A Pedagogical View », dans Aspects of Schenkerian Theory, éd. par David Beach (New Haven : Yale University Press, 1983), 1-38 ; John Rothgeb, « Schenkerian Theory : Its Implication for the Undergraduate Curriculum », Music Theory Spectrum 3 (1981) : 142-49; Christopher Lewis, « Beginning Harmony : The Post-Schenkerian Dilemma », Revue de musique des universités canadiennes, $\mathrm{n}^{\circ} 9 / 1$ (1988) : 136-56.

5 Nous avons choisi d'opter pour la terminologie américaine, plutôt que pour celle mise de l'avant par Célestin Deliège, parce que l'essentiel de la recherche en analyse schenkérienne, donc des références utilisées en classe de même que dans notre propre recherche, sont en langue anglaise. La terminologie de Deliège, première contribution substantielle à la dissémination des idées de Schenker sur la scène musicologique de langue française, a toutefois l'avantage de dissiper la confusion entre un niveau de structure donné et son contenu. Au sujet des problèmes de terminologie dans l'œuvre de Schenker, voir Wallace Berry, « On Structural Levels in Music », Music Theory Spectrum 2 (1980) : 19-45. 
manifestées à l'égard de sa théorie. Par ailleurs, les idées préconçues et les erreurs d'interprétation des concepts fondamentaux qui, en l'absence d'un débat ouvert, tiennent lieu d'explication de la théorie, contribuent grandement à freiner le développement ne serait-ce que d'une curiosité pourl'homme et ses réalisations. L'exemple le plus probant à cet égard est la conception que se font les non-initiés de la structure fondamentale (Ursatz) ${ }^{6}$. Un préjugé tenace présente celle-ci comme l'équivalent d'un paradigme totalement dissocié du discours musical. Schenker définit la structure fondamentale comme la combinaison de la ligne fondamentale (Urlinie) et de l'arpège de la basse (Bassbrechung) (exemple 1). Qu'une structure en apparence aussi simple puisse expliciter l'ensemble des chefs-d'œuvre du répertoire tonal est très souvent perçu comme une aberration. Le profane est convaincu du caractère arbitraire de la pratique schenkérienne : tous les choix analytiques étant à ses yeux assujettis à la nécessité de corroborer l'existence de la structure fondamentale.

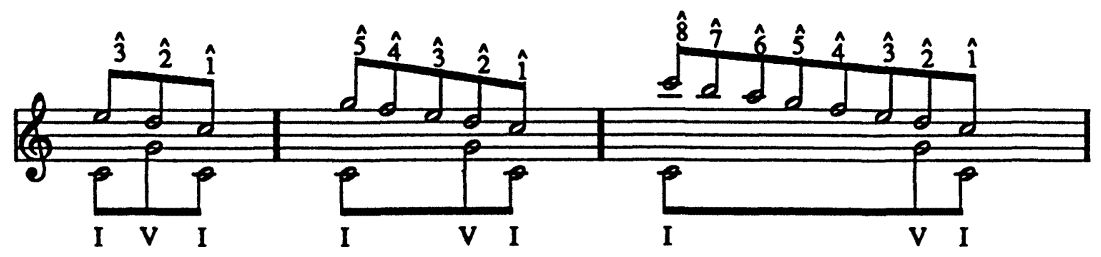

Exemple 1 : Structure fondamentale.

Peut-être devrions-nous poser la question de la quasi complète absence de la théorie schenkérienne des institutions d'enseignement de langue française en termes pragmatiques et nous demander en quoi une théorie musicale complexe, présentée de manière rébarbative par son auteur, exigeant de ses adhérents de

6 Pour une introduction au concept de l'Ursatz, voir Schenker, Free Composition, 3-21. Pour un aperçu des problèmes inhérents à la présentation du concept de l'Ursatz par Schenker, voir Gregory Proctor et Herbert L. Riggins, «Levels and the Reordering of Chapters in Schenker's Free Composition », Music Theory Spectrum 10 (1988) : 102-26. Voir aussi les excellentes recensions de Free Composition par Carl Schachter, «A Commentary on Schenker's Free Composition », Journal of Music Theory 25, $\mathrm{n}^{\circ} 1$ (1981) : 115-42; et Edward Laufer, "Free Composition (Der Freie Satz) by H. Schenker », Music Theory Spectrum 3 (1981) : 158-84. L'évolution du concept de l'Ursatz dans les écrits de Schenker est retracée, dans une approche à la fois succinte et très claire, par Oswald Jonas, Introduction to the Theory of Heinrich Schenker, 129-41. Le lecteur qui s'intéresse aux fondements philosophiques de l'Ursatz pourra consulter William Pastille, « Heinrich Schenker, Anti-Organicist », 19th-Century Music 8 (1984) : 2936 ; idem, «Ursatz : The Musical Philosophy of Heinrich Schenker » (thèse de doctorat, Cornell University, 1985) ; Ruth Solie, « The Living Work : Organicism and Musical Analysis », 19thCentury Music 4 (1980) : 147-56 ; et Kevin Korsyn, « Schenker and Kantian Epistemology », Theoria 3 (1983) : 1-57. 
nombreuses années d'étude et de pratique, peut intéresser professeurs d'analyse musicale et étudiants. Nous ne teitterons pas de répondre à cette question en offrant une réflexion d'ordre épistémologique sur les fondements de la théorie ; plutôt, nous mettrons en évidence les aspects de la théorie schenkérienne qui la distinguent des théories tonales antérieures et qui représentent des acquis substantiels pour l'étude de la tonalité.

\section{Les concepts fondamentaux}

Remarquons tout d'abord que, pour la première fois dans l'histoire de l'analyse de la musique tonale, nous avons affaire à un système dans lequel l'approche structurale de la syntaxe permet de véritablement dépasser la simple « description », soit un exercice taxinomique où la prolifération des catégories considérées échappe à tout principe unificateur. Le concept de niveau de structure (Schicht), de l'interdépendance des différents niveaux (Verwandlungs-Schichten) et, finalement, les concepts de prolongation et de projection compositionnelle (Auskomponierung) que nous définirons plus bas, constituent des outils d'analyse extrêmement puissants. La force et la portée de ces concepts fondamentaux permettent enfin à l'analyste de dépasser le commentaire superficiel, de sonder, puis de rendre compte avec justesse de la complexité des œuvres tonales. L'intérêt de la théorie schenkérienne découle de l'abondance des relations qu'elle permet de révéler entre les éléments particuliers d'une œuvre et cette œuvre prise dans sa totalité.

\section{La notation schenkérienne}

Schenker conçoit la musique sous forme d'un organisme vivant au sein duquel chaque son révèle sa filiation à la structure fondamentale et, simultanément, sa participation individuelle à un niveau de structure spécifique. L'analyse schenkérienne s'applique à démontrer le processus continu d'intégration des différents niveaux de structure dans la création de l'organisme tonal. Cette conception cinétique de la musique en tant que phénomène en constante transformation résiste à la description verbale. Là où le commentaire en prosenécessairement séquentiel puisqu'il ne peut expliquer ou décrire qu'un seul phénomène à la fois-se fait simplificateur, le graphe schenkérien réussit à illustrer les relations multidimensionnelles en jeu dans le discours musical. Cette méthode de notation hiérarchique, l'une des contributions les plus dynamiques de Schenker, constitue donc un outil précieux pour l'enseignement de l'analyse musicale. De plus, amorcé antérieurement et développé systématiquement par Schenker, le phénomène de l'emploi de symboles musicaux pour illustrer 
l'organisation hiérarchique d'une œuvre a eu un formidable impact sur l'évolution de la discipline de l'analyse, tant de la musique tonale que de la musique du $\mathrm{XX}^{\mathrm{e}}$ siècle ${ }^{7}$.

\section{Contrepoint versus composition}

Schenker a abondamment critiqué l'approche qui limite l'enseignement du contrepoint au développement de l'aptitude à pasticher le « style » polyphonique. Encore à ce jour, dans plusieurs conservatoires et facultés de musique, l'enseignement du contrepoint correspond à l'apprentissage de règles d'écriture dont le but est de reproduire les techniques de composition polyphonique en usage au $\mathrm{XVI}^{\mathrm{e}}$ et au XVII ${ }^{\mathrm{e}}$ siècles, telles que l'imitation, le canon et la fugue. Une des contributions majeures de la théorie schenkérienne est d'avoir établi une distinction fondamentale entre les principes régissant la conduite des voix et les règles du contrepoint en tant que techniques de composition. Cette extraordinaire mise au point aura permis d'épurer la pensée contrapuntique des éléments de style qui en dénaturaient l'essence et, ainsi, de réhabiliter la dimension horizontale longtemps occultée par la primauté de la dimension verticale.

En effet, l'approche stylistique du contrepoint n'a rien en commun avec les règles strictes de conduite des voix synthétisées par Johann Joseph Fux à l'époque baroque et revues par Schenker ${ }^{8}$, lesquelles sont, comme l'affirme ce dernier, à la base de tout processus tonal : « Les phénomènes de la composition libre [...] doivent être invariablement compris uniquement en tant que prolongations des principes [contrapuntiques fondamentaux] ${ }^{9}$. " Schenker conçoit les espèces fuxiennes sous forme d'outil pédagogique dont le but, "plutôt que l'enseignement d'un style spécifique de composition, est de guider l'oreille de l'étudiant sérieux pour la première fois dans le monde infini des problèmes musicaux fondamentaux ${ }^{10}$.» Le principe selon lequel les composantes

7 Sur les ramifications de la méthode de notation hiérarchique, voir V. Kofi Agawu, « Schenkerian Notation in Theory and Practice ", Music Analysis 8, $\mathrm{n}^{\circ} 3$ (1989) : 275-301; et Herbert L. Riggins, « Heinrich Schenker's Graphic Notation and Contemporary Variants » (thèse de doctorat, University of Texas Austin, 1981).

8 Schenker, Neue musikalische Theorien und Phantasien II : Kontrapunkt 1 : Cantus firmus und zweistimmigerSatz(Vienne :UniversalEdition, 1910); Kontrapunkt 2:Drei-undmehrstimmiger Satz (Vienne : Universal Edition, 1922) ; traduction anglaise sous le titre de Counterpoint, éd. par John Rothgeb et trad. par John Rothgeb et Jürgen Thym (New York : Schirmer Books, 1987).

9 «The phenomena of free composition [...] are invariably to be understood only as the prolongations of the [fundamental contrapuntal] principles. » Schenker, Counterpoint, 1:13. Toutes les traductions sont de l'auteure d'après les éditions anglaises.

10 « The purpose of counterpoint, rather than to teach a specific style of composition, is to lead the ear of the serious student for the first time into the infinite world of fundamental musical problems. » Schenker, Counterpoint, 1:10. 
structurelles sous-jacentes à la composition libre (freier Satz) sont essentiellement dérivées des lois du contrepoint rigoureux dans la tradition du Gradus ad Parnassum de Fux ${ }^{11}$, constitue l'un des principaux fondements de la théorie schenkérienne $^{12}$. Dans la troisième partie de cet article, nous traiterons de l'importance du contrepoint d'espèces dans la théorie schenkérienne.

\section{L'harmonie : « un univers purement spirituel »}

La théorie de la cohérence organique est fondée sur une conception originale de l'harmonie qui possède le mérite de clarifier la nature de l'articulation du paramètre horizontal (contrepoint) et du paramètre vertical (harmonie) dans les œuvres tonales. Dans la préface à son traité $\mathrm{d}^{\prime}$ harmonie ${ }^{13}$, Schenker précise que, contrairement à la théorie du contrepoint, la théorie de l'harmonie lui apparaît comme « un univers purement spirituel, un système de forces en parfait mouvement, issues de la Nature ou de l'art ${ }^{14}$. » La vision schenkérienne de l'harmonie repose sur une interprétation du degré harmonique (Stufe) en tant qu'idée pure ou concept qui se réalise au moyen de la projection compositionnelle (Auskomponierung). La réalisation du degré harmonique implique que ce dernier peut certes se présenter sous la forme de l'accord, mais aussi et surtout sous la forme des multiples substitutions et transformations de cet accord telles que prescrites par les règles de la conduite des voix et de la basse chiffrée. Schenker définit le degré harmonique comme suit :

Le degré harmonique est une entité plus élevée et plus abstraite [que l'accord]. À l'occasion, il peut même renfermer plusieurs harmonies, dont chacune pourrait être considérée individuellement comme triade ou accord de septième indépendant; en d'autres mots : même si, dans certaines circonstances, certaines harmonies ressemblent à des triades ou accords de septième indépendants, elles peuvent quand même constituer, ensemble,

11 Johann Joseph Fux, Gradus ad Parnassum (Vienne, 1725), trad. et éd. par Alfred Mann sous le titre de The Study of Counterpoint from Johann Joseph Fux's " Gradus ad Parnassum 》 (New York: W. W. Norton, 1971).

12 Au sujet de l'évaluation critique du traité de Fux par Schenker, voir Hellmut Federhofer, « Fux's Gradus ad Parnassum as Viewed by Heinrich Schenker », Music Theory Spectrum 4 (1982): 66-75.

13 Schenker, Neue musikalische Theorien und Phantasien I: Harmonielehre (Vienne : Universal Edition, 1906) ; traduction anglaise sous le titre de Harmony, avec introduction et notes par Oswald Jonas, trad. par Elisabeth Mann Borgese (Chicago : The University of Chicago Press, 1954).

14 "In contrast to the theory of counterpoint, the theory of harmony presents itself to me as a purely spiritual universe, a system of ideally moving forces, born of Nature or of art. " Schenker, Harmony, xxv. 
une seule triade, c'est-dire do-mi-sol, et elles devraient être intégrées au concept de cette triade sur do conçue en tant que degré harmonique. Le degré harmonique impose son caractère plus élevé et plus général en renfermant ou en résumant les phénomènes individuels et en rassemblant leur unité intrinsèque en une seule triade ${ }^{15}$.

Les « phénomènes individuels » mentionnés ici par Schenker représentent les principes de conduite des voix et de la basse chiffrée, ceux-ci étant précisément compris en tant que moyens de réalisation du degré harmonique, tel que mentionné ci-dessus. Cette conception originale de l'harmonie est à la base du développement des concepts de projection compositionnelle (Auskomponierung) et de prolongation, éléments fondamentaux de la pensée schenkérienne. Dans la projection compositionnelle, les principes de conduite des voix, d'origine contrapuntique-donc linéaires et appartenant essentiellement au déroulement horizontal de l'œuvre - constituent les moyens par lequel l' accord, entité verticale d'origine harmonique (Stufe), se déploie dans le temps. Pour définir le concept de prolongation, nous empruntons ici l'excellente définition offerte par John Rothgeb dans les notes éditoriales du traité Counterpoint :

La notion de prolongation occupe une place fondamentale dans la pensée de Schenker. Ce concept très général s'applique à la relation entre les phénomènes simples et leur dérivés plus complexes ; ainsi, tout comme les successions complexes de sons sont dérivées de successions simples au moyen de la Prolongation, les lois complexes d'organisation des sons sont elles aussi dérivées de lois simples par le biais de la Prolongation au sens (plus figuratif) de ramification, extension et adaptation à des situations plus complexes ${ }^{16}$.

15 « The scale-step is a higher and more abstract unit. At times it may even comprise several harmonies, each of which could be considered individually as an independent triad or seventhchord ; in other words : even if, under certain circumstances, a certain number of harmonies look like independent triads or seventh-chords, they may nonetheless add up, in their totality, to one single triad, e.g., C-E-G, and they would have to be subsumed under the concept of this triad on $\mathrm{C}$ as a scale-step. The scale-step asserts its higher or more general character by comprising or summarizing the individual phenomena and embodying their intrinsic unity in one single triad. » Schenker, Harmony, 139.

16 «This concept is fundamental to Schenker's thought. Highly general, it pertains to the relationship between simple phenomena and their more complex derivatives ; thus, just as complex tone-successions are derived from simple ones by Prolongation, so also are complex laws of setting tones derived from simple ones by Prolongation in the (more figurative) sense of ramification, extension, and adaptation to more elaborate situations. » Schenker, Counterpoint, $1: 348 \mathrm{n} 8$. 
La prolongation incarne donc le lien de dépendance réciproque entre les structures sous-jacentes (c'est-à-dire les progressions contrapuntiques fondamentales présentées dans le contrepoint d'espèces) et leur apparition sous une forme élaborée dans la composition libre.

En reconnaissant au principe contrapuntique son rôle premier de prolongation et de projection du degré harmonique, Schenker a redonné aux disciplines du contrepoint et de la basse chiffrée, telle que codifiée par Carl Philipp Emanuel Bach, leur pleine signification dans l'apprentissage de la musique tonale ${ }^{17}$, dans l'apprentissage de la musique tonale. Il va sans dire que l'approche schenkérienne de la structure musicale va àl'encontre de la conception verticaliste de l'harmonie, héritage de la théorie de la basse fondamentale de Rameau. Schenker a traité de l'influence de la pensée de Rameau sur l'évolution de la théorie et de la composition en termes plus ou moins virulents selon le contexte dans lequel apparaît son évaluation critique ${ }^{18}$. L'objection principale de Schenker vis-à-vis de la théorie de Rameau réside dans la primauté accordée à la verticalité de l'écriture au détriment de la dimension horizontale, primauté qui, selon Schenker, serait à l'origine des conceptions divergentes de l'harmonie qui opposent la tradition allemande et la tradition française. Jonas a bien résumé la position de Schenker à ce sujet :

Le concept de Rameau était beaucoup trop étroit [...] ; [sa théorie] était de toute évidence inadéquate lorsqu' appliquée aux finesses de la conduite des voix dans les œuvres de Bach, telles que présentées par C.P.E. Bach dans son Essai [...] Pour [Rameau], « accord » et « degré harmonique » étaient identiques. Il réduisait toute simultanéité de sons à sa position fondamentale supposée et déchirait la délicate texture de la conduite des voix en lanières d'accords plus ou moins intimement reliés entre eux. L'accord, dont le sens et la logique contextuelle dépendent de la conduite des voix, est désormais une entité isolée, généralement sans référence à ce qui précède et à ce qui suit. Chaque note de la ligne de basse, signifiante en tant que

17 Carl Philipp Emanuel Bach, Versuch über die wahre Art das Clavier zu spielen, $2^{\mathrm{e}}$ partie (Berlin, 1762), trad. et éd. par William J. Mitchell sous le titre de Essay on the True Art of Playing Keyboard Instruments (New York : W. W. Norton, 1949).

18 Voir Schenker, dans Harmony, 180, 204 ; idem, préface au traité Counterpoint, 1:xxviii-xxix ; et dans Sylvan Kalib, « Rameau or Beethoven ? Paralytic Standstill or Ingenious Life in Music?, dans « Thirteen Essays from the Three Yearbooks Das Meisterwerk in der Musik by Heinrich Schenker: An Annotated Translation », 3 vol. (thèse de doctorat, Northwestern University, 1973), 3:514. Voir aussi Harald Krebs, « Schenker's Changing View of Rameau : A Comparison of Remarks in Harmony, Counterpoint, and 'Rameau or Beethoven?' ", Theoria : Historical Aspects of Music Theory 3 (1983) : 59-72. 
Auskomponierung d'un accord [...] est alourdie par le poids de la «basse fondamentale » qui inhibe et finalement arrête son mouvement. Toute la vitalité de la musique se fige $[\ldots]$

En termes généraux, la grande erreur de Rameau a été d'interpréter harmoniquement, ou verticalement, une basse qui était composée horizontalement, selon des principes contrapuntiques [...] L'erreur fondamentale de sa doctrine a été de réduire cette ligne de basse vivante à la soi-disant $«$ basse fondamentale $»^{19}$.

L'enseignement de l'harmonie dispensé encore à ce jour dans de nombreuses institutions de langue française et fondé sur l'héritage de Rameau assimile l'identification du statut d'un accord à l'attribution mécanique d'un chiffre romain, sans tenir compte du contexte dans lequel cet accord évolue. L'approche verticale permet ainsi de déclarer deux accords identiques lorsque leurs fondamentales sont identiques. Une des principales sources de plaisir en analyse schenkérienne réside précisément dans la possibilité de reconnaître des significations différentes à des accords pourtant identiques par leur configuration ; enfin, dans la possibilité, en se référant au contexte et au comportement de chaque entité verticale dans ce contexte, d'élucider les relations existant entre ces entités et d'identifier les fonctions qu'elles engendrent.

Nous croyons que les exercices stériles-tels que la pratique de la modulation perpétuelle qui ne s'enracine dans aucune structure organiquement cohérente, la composition de fugues d'école selon des règles et des exceptions multiples trop rarement justifiées du point de vue de la tonalité, ou encore l'application totalement indifférenciée de chiffres romains à toute structure verticale indépendamment du contexte-constituent des approches pédagogiques dépassées. Notre expérience d'enseignement nous permet d'affirmer que

19 « Rameau's concept was much too narrow [...] ; [his theory] most certainly was inadequate if applied to the finesses of voice-leading in the works of Bach as understood in the theories of C.P.E. Bach's Essay [...] For [Rameau] "chord" and "scale-step" were identical. He reduced any simultaneity of tones to its supposed root position and rent the artful texture of voice-leading into strips of more or less closely related chords. The chord, endowed by voice-leading with its full meaning and contextual logic, now was to stand in isolation, generally without reference to what preceded and what followed. The bass line, meaningful as the Auskomponierung of a chord [...] was weighted down, note for note, by the burden of the "ground bass," which inhibited and finally arrested its motion. All the life of music was congealed [...] / In broadest terms, Rameau's great error was to interpret harmonically, or vertically, a bass that was composed horizontally, according to contrapuntal principles [...] To reduce this living bass line to the so-called "ground bass" was the fundamental error of Rameau's doctrine. » Jonas, Introduction à Schenker, Harmony, xi. 
Schenker, le polémiste véhément qui dénigrait l'enseignement de l'harmonie et du contrepoint tel qu'il se faisait dans les conservatoires à son époque, séduit les jeunes musiciens désireux d'échapper à l'aridité d'un apprentissage totalement dissocié de la musique. Que la pratique de l'analyse schenkérienne ramène constamment le musicien à son matériau privilégié, le son, est en quelque sorte une garantie d'authenticité pour les étudiants qui opposent un refus obstiné à une approche désincarnée de leur art.

\section{Conclusion}

Nous terminerons cette première partie en rappelant que les œuvres tonales constituent encore, en cette fin de siècle, la musique la plus fréquemment jouée, écoutée et appréciée, ce qui se traduit par la fréquentation des concerts, les visites chez les disquaires et l'écoute d'émissions radiophoniques. De plus, la grammaire tonale continue d'être largement utilisée dans différents milieux de création, que l'on pense aux musiques entendues au théâtre et au cinéma ou, d'une façon plus mercantile, à l' « industrie » de la musique populaire. Il n'y alors aucun besoin de parler de la nécessité d'un enseignement de la grammaire tonale dans un contexte où les étudiants seront appelés à interpréter, à composer et à enseigner la musique tonale.

Dans un premier temps, ce n'est pas tant la théorie schenkérienne prise dans sa totalité que la conception schenkérienne de l'enseignement de l'harmonie que nous souhaitons voir adopter par le responsable de la formation théorique des musiciens qui fréquentent l'université au niveau du baccalauréat ; et ce, parce que nous la croyons apte à lui fournir un ensemble de concepts et de principes fondamentaux dont la rare intelligibilité ne peut que lui faciliter l'enseignement de la grammaire tonale. Nous citerons, à l'appui de cette proposition, le musicologue belge Célestin Deliège, qui se dit convaincu que la théorie schenkérienne «devrait être à l'origine de progrès considérables au plan pédagogique », et que «non seulement elle s'impose en matière d'analyse [...] mais encore [qu'] elle devrait améliorer considérablement la technique d'écriture de l'étudiant en lui révélant la rationalité du système et en l'amenant à dégager les patterns de base enfouis dans les contextes les plus riches et les plus complexes $^{20}$.»

Dans le cadre des études supérieures et de la recherche, la théorie schenkérienne offre, comme aucune autre théorie tonale antérieure, de puissants outils qui permettent de sonder le système tonal. Elle constitue un modèle flexible qui a ouvert de nombreuses avenues de recherche, comme le confirment les diverses 
adaptations et multiples applications de la théorie, ce dont témoigne l'abondante littérature sur le sujet ${ }^{21}$. Force est de constater qu'il n'existe à l'heure actuelle aucune alternative sérieuse pouvant servir d'assise à l'étude de la syntaxe tonale. On ne peut dès lors que souhaiter l'adhésion des théoriciens et des musicologues de langue française aux idées de Schenker : adhésion non pas à une doctrine mais à un langage commun à tous les chercheurs, afin qu'émerge un discours qui, tout en reflétant la spécificité des préoccupations de chacun, soit susceptible d'engendrer des échanges féconds.

\section{Évaluation des difficultés}

Nous évaluerons ci-dessous les difficultés inhérentes à la diffusion des idées de Schenker en milieu universitaire, difficultés que nous avons nous-mêmes rencontrées dans notre enseignement tant au premier qu'au deuxième cycle.

\section{La complexité de l'œuvre de Schenker}

L'envergure et la profondeur de l'œuvre de Schenker excluent tout espoir d'accès facile à sa pensée. Certes, les professeurs d'analyse musicale peuvent consulter les excellentes traductions anglaises de ses écrits ${ }^{22}$. En revanche, la difficulté bien réelle du propos, ce dont nous reparlerons, les rend inaccessibles aux débutants. Nous savons aussi qu'il est impossible d'accéder à la maîtrise de la théorie de Schenker par la seule lecture de ses ouvrages. De plus, l'évolution graduelle de ses idées-présentées en germe dans Harmonielehre (1906) et développées sur une période de plus de 20 ans dans les publications successives jusqu'à leur formulation définitive dans Der freie Satz (1935)-complique davantage la tâche, étant donné que Schenker, préoccupé par ses travaux en cours, n'a jamais révisé ses ouvrages. Il s'ensuit que certaines des idées exposées dans les ouvrages précédant Derfreie Satz risquent, en raison de leur formulation

21 Voir David Beach, «A Schenker Bibliography », Journal of Music Theory 13 (1969) : 2-37, réimpression dans Readings in Schenker Analysis, éd. par Maury Yeston (New Haven : Yale University Press, 1977); idem, "A Schenker Bibliography : 1969-1979 », Journal of Music Theory 23, $\mathrm{n}^{\circ} 2$ (1979) : 275-86 ; idem, " The Current State of Schenkerian Research », 275307 ; idem, "Schenkerian Theory », Music Theory Spectrum 11, nº 1 (1989) : 3-14.

22 Pour la liste complète des écrits et des traductions des publications de Schenker, voir Beach, « The Current State of Schenkerian Research », 299-301 ; idem, " Schenkerian Theory », 8 ; et Nicholas Rast, " A Checklist of Essays and Reviews by Heinrich Schenker », Music Analysis 7, $\mathrm{n}^{\circ} 2$ (1988) : 121-32. Pour une liste des œuvres analysées par Schenker, voir Larry Laskowski, Heinrich Schenker: An Annotated Index to His Analyses of Musical Works (New York: Pendragon Press, 1978). 
incomplète, de jeter la confusion dans l'esprit des étudiants. Le sujet est en soi complexe et Schenker ne s'est pas soucié de l'élégance de la formulation. Sa prose est obscure, extrêmement dense et, à certains égards, alambiquée. Notons que les traductions en langue anglaise possèdent le mérite de combler certaines lacunes quant à la formulation des concepts fondamentaux par Schenker, souvent très laconique, spécialement en ce qui concerne Der freie Satz. Il va sans dire que cet effort d'interprétation et le haut niveau de qualité de la plupart des traductions facilitent l'accès à l'ensemble de la théorie.

\section{La pratique schenkérienne : conditions préalables}

Tout praticien des techniques d'analyse schenkérienne en conviendra, la connaissance approfondie du contrepoint d'espèces, de la basse chiffrée et de l'harmonie - cette dernière étant, bien entendu, enseignée dans une perspective linéaire-constitue la première condition de développement de la pratique schenkérienne. En effet, l'application de la notation hiérarchique, étape réservée aux études avancées, ne peut être amorcée avec les étudiants qui ne maîtrisent pas les préalables énumérés ci-dessus, apprentissages qui se font au premier cycle universitaire. Or, il est, sinon impossible, à tout le moins extrêmement difficile d'initier à l'analyse schenkérienne tout étudiant dont la formation en harmonie est le résultat d'une approche verticale. Le passage d'une approche verticale à une approche linéaire engendre beaucoup de confusion dans l'esprit des étudiants qui, en général, sont réfractaires aux difficiles réajustements exigés. Ceci étant posé, nous dirons que l'absence d'un traité d'harmonie à teneur schenkérienne, en langue française, constitue l'un des principaux obstacles à la diffusion des idées de Schenker en milieu universitaire francophone.

\section{La structure intermédiaire (Mittelgrund)}

L'exploration du ou des niveaux intermédiaires de structure (Mittelgrund) ${ }^{23}$ d'une œuvre reste en général hors de portée de la majorité des étudiants pour des raisons évidentes. En premier lieu, on ne dispose pas, dans le cadre des programmes, du temps nécessaire au développement de l'expertise requise pour

23 Au sujet des niveaux de structure, de la terminologie utilisée pour les désigner, de leur contenu et de leur nombre, voir Proctor et Riggins, « Levels and the Reordering of Chapters in Schenker's Free Composition », 102-26. Sur le nombre de niveaux de structure en général, Schenker observe dans Free Composition, 26: « Il est impossible de généraliser quant au nombre de niveaux de structure, quoique le nombre puisse être spécifié exactement pour chaque cas individuel $[\ldots]$ » («It is impossible to generalize regarding the number of structural levels, although in each individual instance the number can be specified exactly [...] »). 
mener à bien cette exploration ; en second lieu, cette exploration représente une difficulté réelle. Le véritable enjeu pour le professeur d'analyse schenkérienne consiste alors à éviter de ne mettre en évidence que l'aspect « réductionniste » de la théorie schenkérienne. Edward Laufer, dans son excellente recension de Free Composition, nous rappelle que « la méthode de Schenker n'est pas du tout une "réduction", comme on le dit souvent avec désinvolture, mais la réduction à une structure profonde $e t$, absolument simultanément et inséparablement, un développement à partir de la structure profonde (général) vers la structure de surface (particulier) ${ }^{24}$.»

L'approche analytique par réductions successives à partir du niveau de surface (Vordergrund) jusqu'à la structure profonde résulte des efforts de systématisation et de formalisation de la pensée schenkérienne accomplis entre autres par Felix Salzer d'une part ${ }^{25}$, et par Allen Forte et Steven E. Gilbert d'autre part ${ }^{26}$. Cette attitude tend à miner la puissance du propos de Schenker en évacuant partiellement la dimension créatrice inhérente à sa méthode d'analyse. Plusieurs mises en garde justifiées ont été émises dans la littérature schenkérienne récente ${ }^{27}$. Michael Musgrave observe avec beaucoup de pertinence : « Schenker lui-même a présenté ses idées en tant que "nouvelles théories et fantaisies". Cependant, aux États-Unis, elles se sont transformées : de spéculation créatrice dans l'esprit de la philosophie allemande qu'elles étaient, elles sont devenues des exercices pratiques pour étudiants du premier cycle ${ }^{28}$. »

Certes, les exercices construits avec l'intention explicite de présenter les concepts fondamentaux dans des contextes musicaux dépouillés sont précieux, et certaines techniques de prolongation se prêtent particulièrement bien à

24 "Schenker's procedure is not at all "reduction," as is often flippantly said, but reduction to a background and absolutely simultaneously and inseparably, growth from a background (general) to the specific foreground (unique). » Laufer, "Free Composition (Der Freie Satz) by H. Schenker », 159.

25 Felix Salzer, Structural Hearing : Tonal Coherence in Music, 2 vol. (New York : Dover Publications, 1962 ; éd. orig., New York : Charles Boni, 1952).

26 Allen Forte et Steven E. Gilbert, Introduction to Schenkerian Analysis (New York: W. W. Norton, 1982).

27 Voir entre autres Agawu, "Schenkerian Notation», 291-92 ; Peter Barcaba, «L'analyse schenkerienne et l'interprétation musicale: pour une lecture artistique de l'œuvre de $\mathrm{H}$. Schenker », Analyse musicale 17 (1989) : 91-104 ; 91 ; et Proctor et Riggins, « Levels and the Reordering of Chapters in Schenker's Free Composition », 102-12.

28 "Schenker himself offered his ideas as "new theories and fantasies", though in passage to America they have become dramatically transformed from creative speculation in the spirit of German philosophy into practical exercices for undergraduates. » Michael Musgrave, recension de Music Analysis in Theory and Practice de Jonathan Dunsby et Arnold Whittall, Music Analysis $8, \mathrm{n}^{\text {os }} 1-2$ (1989) : 183-86; 185. 
l'apprentissage par exercices. Nous le verrons dans la troisième partie de cet article. Nous avons pu constater dans le cadre d'un cours d'introduction d'une durée de 13 semaines que la majorité des étudiants réussissent à maîtriser, précisément à l'aide d'exercices, les concepts nécessaires à l'analyse des phénomènes qui appartiennent à la structure de surface des œuvres ou extraits d'œuvres à l'étude. Il est important de reconnaître qu'à ce stade de l'analyse le recours au raisonnement déductif permet à l'étudiant de « réduire », il est vrai, les successions de sons apparaissant dans la musique à des ensembles plus restreints, c'est-à-dire les sons qui « représentent » une structure tonale cohérente $^{29}$. L'étudiant développe alors certaines aptitudes intellectuelles dont la maîtrise d'un langage, soit l'ensemble des règles de la grammaire tonale, et la capacité de raisonner rigoureusement en fonction de l'exercice de déduction qui lui est demandé.

L'exploration de la structure intermédiaire et de la structure fondamentale s'appuie sur l'induction. Le raisonnement de type inductif, qui permet de construire des objets abstraits pour représenter une structure complexe (l'œuvre dans notre cas) est un processus obscur et mal codifié. Sans doute parce qu'il fait appel à l'intuition, mais aussi parce qu'il exige de l'analyste qu'il remonte de l'œuvre aux structures contrapuntiques abstraites sous-jacentes; en d'autres mots, qu'il identifie pour chaque passage de la musique à quel ensemble de structures contrapuntiques abstraites ce passage appartient. L'analyste doit donc ouvrir une voie nouvelle pour chacun des problèmes à résoudre, et, très souvent, la justesse d'une solution dans une analyse de ce niveau ne peut être corroborée qu'intuitivement.

Au contraire de l'approche déductive et du recours à l'apprentissage par les exercices pratiques qui comportent le risque de ne donner aux étudiants que la capacité de manipuler l'enveloppe ou l'aspect externe d'une idée plutôt que son essence, sa forme interne, le raisonnement inductif développe un type d'habileté intellectuelle précieux dans la formation des étudiants, habileté que ces derniers semblent de plus en plus lents à maîtriser. Le développement du raisonnement inductif, jadis pris en charge par des activités formatrices telles que la dissertation philosophique ou l'étude des mathématiques, a en effetété considérablement dévalorisé durant les 20 dernières années. L'étude de l'analyse schenkérienne comble une sérieuse lacune dans la formation d'étudiants qui n'ont que très peu de références aux objets abstraits. Les visées didactiques de Schenker vont malheureusement à l'encontre de la tendance à ne valoriser que très peu ce type

29 Pour un historique du concept de réduction en analyse musicale, voir Robert P. Morgan, "Schenker and the Theoretical Tradition : The Concept of Musical Reduction », College Music Symposium 18 (1978) : 72-96. 
de formation dans les programmes universitaires tels qu'ils existent présentement. Nous sommes forcés de constater que l'étude exhaustive de l'interpénétration des différents niveaux de structure ne peut être entreprise à l'université qu'au doctorat. Toutefois, au risque d'évacuer la part de créativité de la pratique schenkérienne, il incombe au professeur de résister à la tentation de simplifier le propos en occultant la dimension d'interpénétration des différents niveaux de structure (Verwandlungs-Schichten) ou en limitant l'expérience à une approche unidimensionnelle ne concernant que la structure de surface (Vordergrund).

\section{Préalables à l'apprentissage des techniques schenkériennes}

Schenker a clairement défini, en quelques phrases, le « plan didactique » à suivre pour accéder à une "compréhension pratique » du concept de cohérence organique $^{30}$. Ce plan propose l'étude du contrepoint rigoureux (selon FuxSchenker), de la basse chiffrée (selon Johann Sebastian et Carl Philipp Emanuel Bach) et de la composition libre (selon Schenker). L'expression « compréhension pratique » cible très exactement la nature de l'apprentissage proposé. Schenker le pédagogue privilégiait l'enseignement privé du piano pour communiquer ses idées théoriques. La réalité universitaire excluant nécessairement l'enseignement individuel au niveau du baccalauréat, nous devons adapter notre approche au contexte pédagogique qui prévaut.

Notre objectif ici n'est pas de proposer une définition de programme universitaire pour l'apprentissage de la théorie schenkérienne. Plutôt, nous nous attacherons à démontrer qu'il est impossible d'accéder à la maîtrise des techniques schenkériennes sans une compréhension solide des lois fondamentales du contrepoint et de l'harmonie. Pour ce faire, nous démontrerons d'abord que la théorie schenkérienne a pour fondement les grands principes contrapuntiques tels qu'énoncés dans le contrepoint d'espèces ; ensuite, nous présenterons les principaux éléments de la conception schenkérienne de l'harmonie.

\section{Contrepoint rigoureux}

Schenker a qualifié le contrepoint d'espèces de «premiers exercices musicogrammaticaux ${ }^{31}$. La valeur pédagogique de ces exercices réside en ce qu'ils permettent aux étudiants d'acquérir une très solide perception auditive (1) des propriétés de chacun des intervalles formés par les voix en mouvement ; (2) des mouvements utilisés pour approcher et quitter les intervalles; et (3) de la 
subordination des dissonances (passage, broderie, retard) aux consonances-en d'autres mots, une « compréhension pratique » du vocabulaire tonal.

Le contrepoint d'espèces nous permet aussi de présenter les consonances et les dissonances indépendamment de leurs propriétés harmoniques. Par exemple, la première espèce ne faisant usage que de consonances, l'étudiant y découvre les divers degrés de stabilité caractérisant l'octave, la quinte juste, les tierces et les sixtes majeures et mineures. La dissonance apparaît pour la première fois dans la deuxième espèce où, avec deux blanches contre une ronde, un élément d'organisation métrique est introduit. La différenciation entre temps fort et temps faible ouvre la voie à l'utilisation de la dissonance qui apparait au temps faible, en note de passage, donc approchée et quittée par mouvement conjoint. Il n'est évidemment pas nécessaire de présenter ici tous les principes contrapuntiques exposés dans les cinq espèces. Nous nous pencherons plutôt sur un principe contrapuntique spécifique, notamment, la note de passage dissonante. Ce choix s'impose pour deux raisons : (1) parce que la note de passage dissonante incarne avec clarté un des fondements du système tonal : la subordination de la dissonance à l'absolue primauté des consonances ; et (2) parce qu'elle est à l'origine du concept de projection compositionnelle, comme nous le verrons plus bas. Nous reprendrons ici la démonstration du rôle de la note de passage dissonante dans l'élaboration du concept de projection compositionnelle par Schenker ${ }^{32}$.

Schenker amorce sa démonstration par l'illustration de la relation entre temps fort et temps faible lorsque, dans un contrepoint à deux voix, les deux blanches sont consonantes. Dans l'exemple 2, la deuxième blanche est conforme à l'harmonie établie au premier temps. La somme des deux blanches, dans chacune des mesures, produit un accord complet ou incomplet clairement identifiable. En termes schenkériens, la technique de projection compositionnelle représentée par la deuxième blanche est celle du déploiement (Ausfaltung) ou expression horizontale d'une entité verticale. Le lecteur observera ici que, lorsque présentée dans le contexte dépouillé du contrepoint d'espèces, cette technique est tout à fait accessible au débutant.

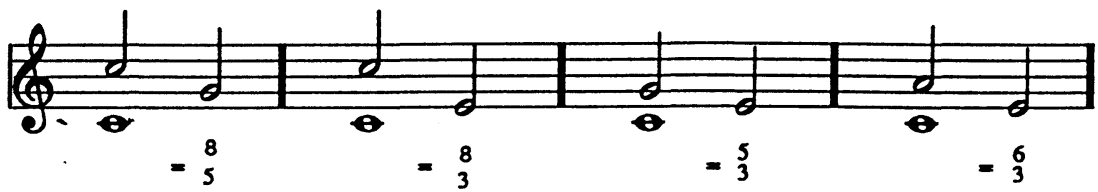

Exemple 2: Blanches fondamentales.

32 Étant donné que Schenker présente cette démonstration au chapitre sur le contrepoint à trois voix, dans le traité Counterpoint, 2:55-58, les propriétés harmoniques des prototypes contrapuntiques 
L'exemple 3a présente une situation légèrement différente. L'arrivée de la deuxième blanche marque un changement à l'intérieur de la mesure : de l'harmonie de do majeur à l'harmonie de la mineur. Dans l'exemple 3b, nous avons affaire à une situation ambiguë. D'une part, nous pourrions concevoir que l'arrivée de la deuxième blanche articule un changement d'harmonie de do majeur à la mineur, l'harmonie de do majeur étant établie par la fondamentale do à la voix inférieure, doublée au contrepoint supérieur. D'autre part, nous pourrions aussi concevoir le $l a$, deuxième blanche, en tant que complément de l'harmonie de la mineur. Cette deuxième hypothèse suppose toutefois que l'harmonie de la mineur est sous-entendue dès l'arrivée de la première blanche sur le temps fort.

a)

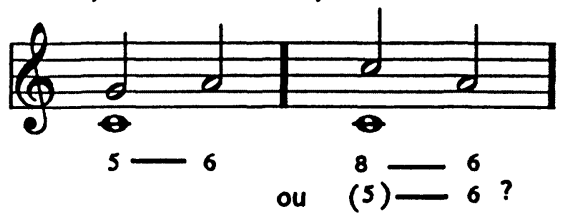

Exemple 3 : Do majeur et $l a$ mineur (3a); do majeur ou la mineur (3b).

Le même type d'ambiguïté surgit lorsque le contrepoint està la voix inférieure. Dans l'exemple 4a, l'effet créé est indéniablement celui d'un accord de do majeur. Toutefois, dans le cas des exemples $4 b$ et $4 c$, on ne peut parler avec certitude d'un changement d'harmonie. Dans le commentaire accompagnant cet exemple, Schenker introduit l'idée d'une représentation mentale d'un son :

l'harmonie du temps fort [...] peut contrôler notre écoute de telle sorte que là où le temps faible le permet, nous imaginons que la première blanche est soutenue-soit qu'elle continue à résonner ; mais le contrepoint rigoureux n'a pas encore le pouvoir d'éliminer le doute qui peut apparaître concernant

étudiés sont prises en considération. Au sujet des avantages qu'il y a à étudier le contrepoint rigoureux avant l'harmonie, voir Jack Adrian, « Pedagogically Speaking : On Studying Species Counterpoint Before Studying Harmony », In Theory Only 8, $\mathrm{n}^{\circ} 2$ (1984) : 15-19. Le deuxième volume de Counterpoint est consacré d'abord à la pratique du contrepoint à trois, quatre et plusieurs voix, puis, dans le chapitre intitulé «Bridges to Free Composition», au mélange d'espèces. Schenker y fait la démonstration de la relation entre les lois fondamentales du contrepoint rigoureux et la composition libre. L'étude de ce dernier chapitre est essentielle à la compréhension de Free Composition, où Schenker expose sa théorie dans sa forme définitive. Sur le rôle de la note de passage dissonante, voir aussi Rothgeb, « Strict Counterpoint and Tonal Theory », Journal of Music Theory 19 (1975) : 260-84; et Jonas, Introduction to the Theory of Heinrich Schenker, 52-62. 
a)

b)

c)

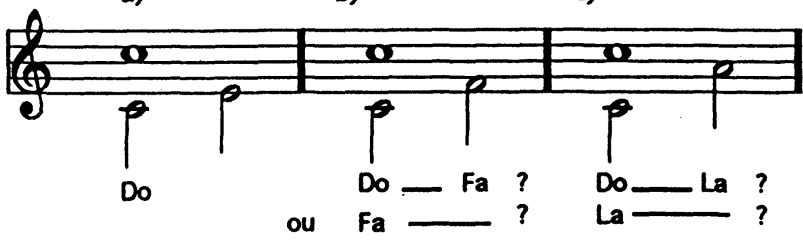

Exemple 4 : Do majeur (4a); do majeur et/ou $f a$ majeur (4b); do majeur et/ou la mineur (4c).

un changement d'harmonie (sauf dans le cas incontestable de 5-6, [notre exemple 3a]), ni le pouvoir de rendre le sens avec précision ${ }^{33}$.

Lorsque, en deuxième espèce, la deuxième blanche est dissonante et, conséquemment, traitée en note de passage reliant deux verticalités consonantes, ce « deuxième intervalle reste encore sous l'influence de l'harmonie du temps fort précédent [...] comme si l'harmonie entendue au premier temps était toujours présente au deuxième temps-comme si elle résonnait toujours ${ }^{34}$. »

Dans le commentaire accompagnant l'exemple 5, Schenker introduit la notion de rétention d'un son par la mémoire musicale, de la capacité de cette dernière à suppléer un son qui n'est pas explicitement présent.

a)

b)

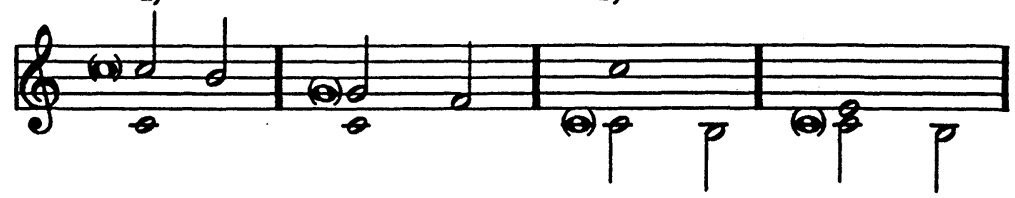

Exemple 5 : Rétention d'un son par la mémoire musicale.

33 « the harmony of the downbeat [...] can control our hearing in such a way that, wherever the upbeat permits, we imagine the first half-note as sustaining - that is, as continuing to sound ; but strict counterpoint does not yet have the power to eliminate the doubt that may arise concerning a change of harmony (except in the indisputable case of 5-6), to say nothing of yielding a complete precision of meaning. » Schenker, Counterpoint, 2:56.

34 « the second interval still remains under the influence of the harmony of the preceding downbeat [...] as if the harmony of the downbeat were still present at the upbeat-as if it were still sounding. » Schenker, Counterpoint, 2:57. 
[Dans l'exemple 5a], du seul fait que la note inférieure est soutenue, le son du premier intervalle consonant se prolonge dans la mémoire de l'auditeur, et ce d'autant plus clairement que toutes les définitions d'intervalles sont basées sur la note la plus basse. Par contre, dans le deuxième cas [exemple $5 b]$, la voix inférieure [...] n'assure plus la continuation audible de l'harmonie établie au temps fort ; par conséquent, il devient nécessaire dans ce cas de garder en mémoire l'harmonie entendue au premier temps. Si, pour clarifier notre propos, nous écrivons à [l'exemple 5b] le son qui doit être prolongé dans un registre inférieur-puisqu'il est de nature purement imaginaire (geistig) [exemple 6] :

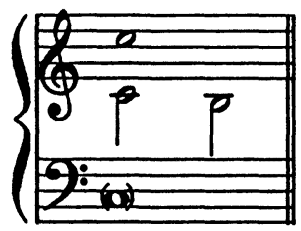

nous avons alors un aperçu de la véritable nature de la voix inférieure [...] et de la façon dont celle-ci reproduit l'effet d'une voix supérieure dans sa relation avec un degré [harmonique (Stufe)], dont le registre est encore plus bas. En d'autres mots, c'est précisément la note de passage dissonante qui confirme l'harmonie entendue au premier temps, et ce, de manière plus sûre et plus absolue qu'un deuxième temps consonant, lequel, comme nous l'avons vu plus haut [voir exemples $3 \mathrm{~b}$ et $4 \mathrm{a}, \mathrm{b}, \mathrm{c}$ ], n'assure pas d'unité d'effet harmonique [à l'intérieur de la mesure] et conduit souvent à un changement plus ou moins explicite d'harmonie ${ }^{35}$.

La note de passage dissonante (temps faible) a donc pour effet de prolonger la consonance du temps fort précédent jusqu'au temps fort suivant. Nous

35 « because at $a$ the lower tone is sustained, that act of sustaining alone prolongs in one's memory the sound of the consonant first interval, and all the more clearly so because all intervallic definitions are based on the lowest voice. In the second case, at $b$, on the other hand, the lower voice gives up the means [...] to provide an audible continuation of the harmony established on the downbeat ; therefore, it is particularly necessary in this case to retain the harmony of the downbeat at least in memory. If, for the sake of greater clarity, we write at $b$ the tone to be prolonged in a lower register-since it is purely imaginary (geistig) in nature - : / then we gain an insight into the true nature of the lower voice [...], and how, in relation to the scale degree conceived as lying still lower, it actually takes on the meaning of another upper voice. In other words, it is precisely the dissonant passing tone that confirms the harmony of the downbeat more reliably and emphatically than the consonant upbeat, which, as we saw earlier, does not imply a unity of harmonic effect [within the bar] and often leads even to a more or less explicit change of harmony. » Schenker, Counterpoint, 2:57. 
trouvons dans cet effet de continuation une réalisation élémentaire de la projection compositionnelle, tel que l'observe Schenker dans le passage suivant :

la première apparition [en contrepoint rigoureux] de la note de passage dissonante produit une curieuse intrusion de l'imaginaire : celle-ci consiste dans la rétention, inaperçue par l'oreille, du point de départ consonant qui accompagne la note de passage dissonante dans sa traversée de l'espace de tierce. Tout comme si la dissonance transportait toujours avec elle l'impression de son origine consonante $[\ldots]$

Les implications de cet effet sont d'une grande importance : nous reconnaissons dans la note de passage dissonante le véhicule le plus sûrà vrai dire le véhicule unique-du contenu mélodique. Alors qu'en première espèce la ligne mélodique se déploie encore laborieusement, son par son, dans la deuxième espèce nous la voyons évoluer à l'intérieur du cadre d'une sonorité verticale de soutien. Ainsi, même le contrepoint à deux voix laisse paraître les débuts de la projection compositionnelle mélodique-soit le déploiement simultané de la même harmonie en direction horizontale et verticale -dans la mesure où le contrepoint à deux voix est capable de créer une relation temps fort-temps faible de sorte que les deux temps expriment la même harmonie. Lorsque le temps faible est consonant, cette relation temps fort-temps faible peut être ou ne pas être présente, selon l'intervalle entendu au deuxième temps; mais elle est toujours présente lorsque le temps faible est dissonant ${ }^{36}$.

Le lecteur observera que la note entendue dans la voix inférieure, le cantus, quoique strictement gouvernée par le mouvement linéaire, se pose en archétype idéal du degré harmonique (Stufe). En effet, cette note individualise, établit

36 « the first appearance [in strict counterpoint] of the dissonant passing tone produces a curious intrusion of the imaginary : it consists in the covert retention, by the ear, of the consonant point of departure that accompanies the dissonant passing tone on its journey through the third-space. It is as though the dissonance would always carry along with it the impression of its consonant origin $[\ldots]$ /The implications of this effect are of great importance : we recognize in the dissonant passing tone the most dependable-indeed the only-vehicle of melodic content. While in the first species the melodic line still unfolds laboriously, sound by sound, in the second species we see it move ahead within the framework of a sustaining vertical sonority. Therefore even twovoice counterpoint shows the beginnings of melodic composing-out-that is, the simultaneous unfolding of the same harmony in both vertical and horizontal directions- to the extent that it is capable of setting up a relationship of downbeat and upbeat such that both express the same harmony. When the upbeat is consonant, this relationship of downbeat and upbeat may or may not be present, depending on the particular interval ; but it is always present when the upbeat is dissonant. » Schenker, Counterpoint, 2:57-58. 
l'identité des notes entendues dans les voix supérieures. Il est particulièrement intéressant de constater que nous avons là une proposition accessible à tout débutant et à partir de laquelle nous pouvons effectuer une première incursion dans la théorie de l'harmonie de Schenker afin d'en présenter les principaux éléments : les concepts de degré harmonique et de niveaux de structure. Avant d'aborder l'harmonie schenkérienne, nous ajouterons que la dissonance traitée en note de passage-soit approchée et quittée par mouvement conjoint, ou, en d'autres mots, porteuse de mouvement, de continuation du mouvement-est à l'origine du concept schenkérien de progression linéaire (Auskomponierungszug). La progression linéaire, ou étalement compositionnel d'un intervalle, est une extension du concept de note de passage. Dans la progression linéaire, la note de passage-qui traverse initialement l'espace de tierce et reprend en ceci les caractéristiques de la ligne fondamentale (Urlinie) —est appliquée aux intervalles de quarte, de quinte, de sixte, de septième et d'octave ${ }^{37}$.

Les observations dégagées ci-dessus illustrent l'influence profonde d'un principe contrapuntique-la note de passage dissonante-sur l'élaboration des concepts à l'origine de la théorie de Schenker, et, conséquemment, l'importance de l'étude du contrepoint d'espèces. En raison de contraintes temporelles, il est impossible d'aborder l'étude du mélange des espèces à trois voix au baccalauréat. Cet apprentissage est toutefois indispensable à quiconque désire approfondir la pensée de Schenker et comprendre sa théorie telle qu'elle est exposée, dans sa forme définitive, dans Free Composition. En effet, comme le fait remarquer Pastille, il ne suffit pas de posséder une solide connaissance des outils d'analyse présentés dans Free Composition pour accéder à la maîtrise de la théorie schenkérienne, car

Free Composition ne renferme pas toute la théorie de Schenker; il en représente seulement le point culminant ; la théorie en soi est solidement enracinée dans les lois fondamentales élaborées dans Counterpoint. Les principes énoncés dans Free Composition sont au reste de la théorie de Schenker ce que les mathématiques pures sont à l'algèbre; on ne peut maîtriser les techniques avancées sans un entendement très clair des lois fondamentales ${ }^{38}$.

37 Pour plus d'information au sujet de la progression linéaire, voir entre autres Schenker, Free Composition, 43-46, 73-82 ; et Jonas, Introduction to the Theory of Heinrich Schenker, 52-89.

38 " Free Composition is not the whole of Schenker's theory ; it is only the apex of the theory, which rests solidly on the basic fundamentals laid out in Counterpoint. The theory of Free Composition is to the rest of Schenker's theory as higher mathematics is to algebra : without a secure grasp of the basics, success with the advanced techniques is impossible. "William Pastille, «Strict Counterpoint and Free Composition: Review/Essay on the Rothgeb/Thym translation of Schenker's Kontrapunkt », Theoria 3 (1988):161-69; 166. 
Avant de conclure, le traité de contrepoint que nous utilisons en classe appelle quelques observations. On ne saurait trop insister sur la valeur pédagogique du traité de Schenker. Toutefois, l'approche hautement spéculative privilégiée par ce dernier se prête difficilement à l'apprentissage par la réalisation de versions, qui caractérise les cours d'écriture. Nous utilisons plutôt Counterpoint in Composition $^{39}$, dont la première partie est conçue pour un programme de baccalauréat. Cet ouvrage présente les cinq espèces à deux et à trois voix telles que codifiées par Fux et repensées par Schenker. Ceci étant posé, il n'en demeure pas moins que l'étude du traité Counterpoint de Schenker constitue la principale voie d'accès à la théorie schenkérienne, plus particulièrement la section sur les mélanges d'espèces intitulée "Bridges to Free Composition », où Schenker expose sa conception de la relation entre contrepoint rigoureux et composition libre, tel que mentionné précédemment (voir note 32 ).

\section{Harmonie}

Schenker ne concevait pas l'harmonie sous la forme de discipline préparatoire à l' analyse, comme l'indique son " plan didactique ", dans lequel n' apparaissent que contrepoint rigoureux et basse chiffrée. Il a écrit un ouvrage sur la basse chiffrée, Generalbasslehre, dont seule l'introduction— « Von der Stimmführung des Generalbasses » («The Voice Leading of Thorough Bass »)—a été publiée quelque temps après sa mort ${ }^{40}$. En l'absence d'un texte complet de Schenker sur la basse chiffrée, une traduction de cet ouvrage restant à paraître ${ }^{41}$, l'étude de sa conception de l'harmonie pose un problème au débutant. Le traité Harmonielehre (1906) a été publié presque 30 ans avant Der freie Satz (1935) et ne peut évidemment constituer la principale référence pour l'étude de l'harmonie, les idées que Schenker y exposent ayant considérablement évolué dans ses écrits subséquents.

Il existe certains manuels, en anglais, que l'on peut qualifier de pré-schenkériens et qui sont bien adaptés au contexte universitaire. Pour l'enseignement de

39 Felix Salzer et Carl Schachter, Counterpoint in Composition : The Study of Voice Leading (New York : McGraw-Hill, 1969).

40 L'introduction est parue dans Der Dreiklang 3 (juin 1937). Toute référence à la basse chiffrée par Schenker se rapporte au Versuch de Carl Philipp Emanuel Bach et à un manuscrit de la main de Bach, publié dans Philipp Spitta, Johann Sebastian Bach (New York : Dover Publications, 1951), 2:315-47 (version intégrale de la traduction de l'allemand par Clara Bell et J. A. FullerMaitland parue en 1889). Pour plus d'information sur Schenker et la théorie de la basse chiffrée, voir Hedi Siegel, «A Source for Schenker's Study of Thorough Bass : His Annotated Copy of J. S. Bach's Generalbassbüchlein », dans Schenker Studies (Cambridge : Cambridge University Press, 1990).

41 Une traduction de Hedi Siegel sera publiée dans The Music Forum (vol. 6, $2^{\mathrm{e}}$ partie). 
l'harmonie au baccalauréat, nous retenons plus particulièrement Harmony and Voice Leading ${ }^{42}$. Sans introduire de techniques schenkériennes spécifiques, les auteurs présentent la grammaire tonale à la lumière de l'interaction du paramètre horizontal et du paramètre vertical, condition essentielle à la compréhension des concepts de prolongation et de niveaux de structure. Largement accepté dans les milieux informés, ce manuel se situe dans le sillage de l'effort de vulgarisation et de dissémination de la théorie schenkérienne entrepris par Felix Salzer dans les années 50. Dans son ouvrage Structural Hearing: Tonal Coherence in Music, Salzer expose les fondements de la pensée harmonique de Schenker plus systématiquement que ne l'a fait ce dernier. Quoique cet ouvrage ne soit pas conçu pour remplacer les écrits de Schenker (de nombreux concepts schenkériens n'y sont pas présentés), il offre une excellente formulation pédagogique de certains principes. Nous nous en inspirons dans la démonstration qui suit en raison des qualités de clarté et de concision de l'exposé. Toutefois, l'effort de systématisation de la pensée harmonique de Schenker entrepris par Salzer a donné lieu à une extension du concept schenkérien de structure. L'émergence d'une nouvelle classification d'accords et de fonctions d'accords ouvre la porte à l'application de la méthodologie schenkérienne au répertoire pré-baroque de même qu'à la musique post-tonale, ramifications qui débordent notre propos ${ }^{43}$. Nous ne retiendrons donc des travaux de Salzer que ce qui est représentatif de la conception schenkérienne de l'harmonie. Après avoir présenté les fondements de la pensée harmonique de Schenker selon Salzer ${ }^{44}$, nous commenterons une série de progressions couramment utilisées dans le répertoire tonal et qui illustrent un type spécifique de prolongation : la prolongation contrapuntique d'un accord structurel.

\section{Fondements de la pensée harmonique de Schenker}

À l'instar de Schenker, Salzer établit une distinction entre les accords d'origine contrapuntique et les accords d'origine harmonique qu'il qualifie, respectivement, d'accords de prolongation et d'accords structurels. Cette distinction repose sur

42 Edward Aldwell et Carl Schachter, Harmony and Voice Leading, $2^{\mathrm{e}}$ éd. (New York : Harcourt, Brace and Jovanovich, 1989).

43 À ce sujet, voir entre autres Milton Babbit, recension de Structural Hearing de Felix Salzer, Journal of the American Musicological Society 5, $\mathrm{n}^{\circ} 3$ (1952) : 260-65; Jonas dans Schenker, Harmony, viii (note 2) ; Joseph N. Straus, « The Problem of Prolongation in Post-Tonal Music », Journal of Music Theory 31 (1987) : 1-21 ; Jonathan Dunsby et Arnold Whittall, Music Analysis in Theory and Practice (Londres : Faber Music, 1988), 53-61 ; et Annie Cœurdevey, « L'analyse schenkerienne et ses frontières », Revue de musicologie 79, $\mathrm{n}^{\circ} 1$ (1993) : 5-30.

44 Salzer, Structural Hearing, 1:7-96. 
la conception schenkérienne des fonctions respectives du contrepoint et de l'harmonie. D'une part, le contrepoint représente la force motrice du mouvement musical. Cette force, qui est l'expression du sens de la direction du mouvement musical, est incarnée par l'intervalle de seconde. D'autre part, l'harmonie engendre la charpente structurelle au sein de laquelle les accords issus du mouvement contrapuntique évoluent. La spécificité purement harmonique de la charpente structurelle est déterminée par l'intervalle de quinte. Ainsi, tout organisme musical est constitué de l'interaction des principaux éléments de la composition, soit « les facteurs de mouvement et de prolongation, qui sont les éléments du contrepoint, et les facteurs de stabilité et de structure, qui sont ici appelés progressions harmoniques ${ }^{45}$."

Salzer qualifie la progression I-V-I de progression harmonique fondamentale. Le lecteur observera que la progression harmonique fondamentale de Salzer est identique à l'arpège de la basse (Bassbrechung) de la structure fondamentale (Ursatz)—une "horizontalisation" incomplète de l'accord parfait majeur, l'accord de la "Nature ${ }^{46}$. La structure fondamentale (Ursatz)—un produit de la nature et de l'art ${ }^{47}$-ne peut être prolongée ; c'est donc au niveau de la structure intermédiaire (Mittelgrund) et de la structure de surface (Vordergrund) qu'apparaissent les possibilités de prolongation de l'arpège de la basse

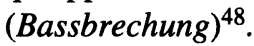

Parmi les possibilités d'élaboration de l'arpège de la basse, ou progression harmonique fondamentale, présentées par Salzer ${ }^{49}$, nous retiendrons en premier lieu l'interpolation d'accords intermédiaires, soit les degrés III, II et IV (figure 1), dans l'espace de quinte ascendante, I-V. Dans la progression I-III-V-I, le troisième degré provient de l'intervalle de quinte arpégé. La tierce qui en résulte (Terzteiler) prendra une signification harmonique lorsqu'elle représente l'accord III, et contrapuntique lorsqu'elle représente le renversement de l'accord de tonique, $\mathrm{I}^{6}$. Dans l'enchaînement I-II-V-I, le deuxième degré revêt aussi une double signification : harmonique en ce qu'il est situé une quinte au-dessus de la dominante, et contrapuntique par la relation de seconde qu'il entretient avec l'accord de tonique. La relation de quinte descendante entre l'accord de tonique et l'accord de sous-dominante affaiblit le statut harmonique du quatrième degré.

45 « factors of motion and prolongation which are elements of counterpoint, and factors of stability and structure which are here called harmonic progressions. " Salzer, Structural Hearing, 1:50.

$46 \mathrm{La}$ théorie de la cohérence organique repose sur le fait que l'accord parfait majeur existe dans la " Nature ", celui-ci étant engendré par la production des troisième et cinquième harmoniques. Schenker expose sa thèse dans Harmony, 3-54.

47 Schenker, Harmony, 11.

48 Schenker, Free Composition, 29-36, 68-73.

49 Salzer, Structural Hearing, 1:89-90. 
Nous ne reprendrons pas ici la thèse formulée par Schenker ${ }^{50}$ sur le rapport de l'accord de sous-dominante aux accords de tonique et de dominante. Nous retiendrons seulement que la distance de seconde entre l'accord de sousdominante et l'accord de dominante témoigne clairement de l'origine contrapuntique de l'enchaînement IV-V.

$\begin{array}{lllll}\text { I } & \text { II } & \text { IV } & \text { I } \\ & & \text { II } & & \\ & \text { IV } & & \end{array}$

\section{Figure 1}

Les accords $\mathrm{II}^{6}$ et VI engendrent des progressions harmoniques secondaires (figure 2). Quoique placé en relation de seconde ascendante à la fondamentale de V, $\mathrm{II}^{6}$ assumera la fonction de IV ou de II selon le traitement des voix intermédiaires (voir $\left.\mathrm{IV}^{(5)-6}\right)^{51}$. L'absence complète de relation de quinte dans l'enchaînement VI-V affaiblit considérablement le statut harmonique de cet enchaînement. Salzer accepte l'accord VI en raison de sa filiation à III ; plus précisément, parce que la progression I-VI(-V) reprend, en mouvement descendant, la progression par tierce I-III(-V $)^{52}$. La progression I-VI-V permet notamment d'approcher la fondamentale de l'accord de dominante par mouvement conjoint descendant. Toutefois, « l'artificialité », pour reprendre l'expression de Salzer, de l'équation I-VI-I-III est responsable du rôle ambigu de VI dans la grammaire tonale. Conséquemment, VI assume régulièrement le statut d'un accord généré par mouvement linéaire, un accord-passage entre I et $\mathrm{V}$.

$\begin{array}{llll}\text { I } & \mathrm{II}^{6} & \mathrm{~V} & \mathrm{I} \\ & \mathrm{VI} & \end{array}$

\section{Figure 2}

Salzer conclut en spécifiant que la progression harmonique fondamentale, ses trois élaborations (figure 1) et les progressions harmoniques secondaires (figure 2) "démontrent le principe harmonique en musique dans sa forme la plus concentrée et la plus pure ${ }^{53}$. » Notons que le caractère harmonique des progres-

50 Schenker, Free Composition, 30-31, 69.

51 Schenker, Free Composition, 33.

52 Salzer, Structural Hearing, 1:90.

53 « demonstrate the harmonic element in music in its most concentrated and purest form. » Salzer, Structural Hearing, 1:90. 
sions présentées ci-dessus n'est en aucune façon altéré par l'introduction de la septième-d'origine essentiellement contrapuntique-dans V, II et IV ${ }^{54}$. Toutefois, nous avons pu observer que les accords interpolés dans la progression $\mathrm{I}-\mathrm{V}$ révèlent leur forte appartenance à la sphère contrapuntique. Comme le fait remarquer Salzer, « alors qu'il est possible de démontrer le principe contrapuntique sans avoir recours à l'influence de l'harmonie, les progressions harmoniques ne sont pas totalement affranchies de caractéristiques contrapuntiques ${ }^{55}$. " En d'autres mots, l'harmonie et le contrepoint sont indissociables et s'inscrivent en perpétuelle interdépendance dans l'organisation du langage tonal. De même, l'identification des accords structurels et des accords de prolongation ne peut être accomplie sans l'analyse de la structure mélodique; et, inversement, l'identification des notes structurelles et des notes de prolongation de la voix supérieure ne peut être effectuée sans que les entités verticales et le mouvement de la basse ne soient pris en considération.

Salzer attribue donc aux progressions présentées ci-dessus le rôle de charpente structurelle harmonique au sein de laquelle évolueront les accords de prolongation, d'origine contrapuntique, au niveau de la structure intermédiaire et de la structure de surface. Tel que mentionné précédemment, l'effort de systématisation de Salzer a donné lieu à une extension du concept schenkérien de structure. Les paramètres harmonie-structure et contrepoint-prolongation ne sont désormais plus les seuls paramètres compositionnels; on assiste à l'émergence de nouveaux paramètres : harmonie-prolongation et contrepoint-structure. En d'autres mots, les accords d'origine harmonique et les progressions harmoniques peuvent désormais assumer une fonction de prolongation, et les accords et progressions d'origine contrapuntique peuvent assumer un rôle structurel ${ }^{56}$. Le paramètre «contrepoint-structure » et son corollaire, l'accord issu du mouvement contrapuntique qui assume une fonction structurelle, n'existent pas comme tel dans la théorie schenkérienne. Conséquemment, nous ne retiendrons, dans la démonstration qui suit, que les paramètres qui sont en conformité avec la conception schenkérienne de l'harmonie.

54 La résolution de la septième par mouvement conjoint descendant et sa préparation, par note de passage dans $\mathrm{V}^{8-7}$ et par note commune dans $\mathrm{II}^{6 / 5}$ et $\mathrm{IV}^{7}$, sont des principes contrapuntiques appartenant aux deuxième et quatrième espèces respectivement.

55 "Although it is possible to demonstrate counterpoint without any harmonic influence, the harmonic progressions show certain contrapuntal characteristics. » Salzer, Structural Hearing, 1:90.

56 Salzer, Structural Hearing, 1:148-62. 


\section{Prolongation contrapuntique d'un accord structurel}

Pour des raisons évidentes d'espace, nous ne traiterons que d'un des types de prolongation dérivés des paramètres harmonie-structure et contrepoint-prolongation proposés par Salzer. Nous avons choisi la prolongation contrapuntique d'un accord structurel parce que la « compréhension pratique » de ce paramètre constitue un préalable essentiel à l'apprentissage des techniques schenkériennes. Ce paramètre est présenté dès la première session d'harmonie, soit après l'étude du contrepoint rigoureux. L'apprentissage de la distinction fondamentale entre accords d'origine contrapuntique et accords d'origine harmonique se fait durant les deux premières années du baccalauréat. L'étudiant qui maîtrise cette distinction est déjà à même d'effectuer une étude relativement approfondie du premier niveau de structure d'une œuvre, tel que nous le verrons dans l'analyse de l'Inventio 12 de Johann Sebastian Bach.

Nous avons sélectionné (1) une série de progressions où des accords issus du mouvement linéaire prolongent les accords des trois grandes catégories syntaxiques de la charpente structurelle : tonique (exemple 7), pré-dominante (exemple 8), dominante (exemple 9); et (2) un modèle d'intervalles qui soit prolonge un seul accord structurel (exemple 10a), soit relie deux accords structurels différents (exemple 10b). L'exemple 10 ne retient que deux des nombreux modèles d'intervalles qui jouent un rôle fondamental dans la prolongation, et qui sont à l'origine des passages séquentiels dont nous reparlerons plus bas. Les exemples 7,8 et 9 juxtaposent une progression réalisée à quatre voix et une réduction analytique. Les chiffres arabes surmontés d'un accent circonflexe ${ }^{(} \wedge$ ) représentent les degrés mélodiques et les crochets ([]) indiquent une dominante secondaire, soit une harmonie autre que l'harmonie de tonique précédée, et à l'occasion suivie, de sa propre dominante. La relation de quinte dominantetonique est alors reproduite, d'où le terme technique «tonicisation ».

La notation utilisée, basse chiffrée et chiffres romains, diffère selon qu'elle accompagne la progression ou la réduction. Dans le cas des progressions réalisées à quatre voix, nous avons placé un chiffre romain sous chaque entité verticale. Les chiffres romains entre parenthèses indiquent que les accords auxquels ils sont associés sont d'origine contrapuntique. Dans le cas des réductions, nous séparons la basse chiffrée et le chiffre romain. L'accolade indique la durée de la prolongation. Nous utilisons cette notation dans nos cours parce qu'elle permet aux étudiants d'associer la basse chiffrée à l'activité contrapuntique et le chiffre romain à l'activité harmonique. En articulant ainsi la différenciation des fonctions, les étudiants sont à même d'inscrire leur analyse dans une perspective hiérarchique et d'acquérir une « compréhension pratique » de la notion de niveau de structure dès la première étape de leur apprentissage. 

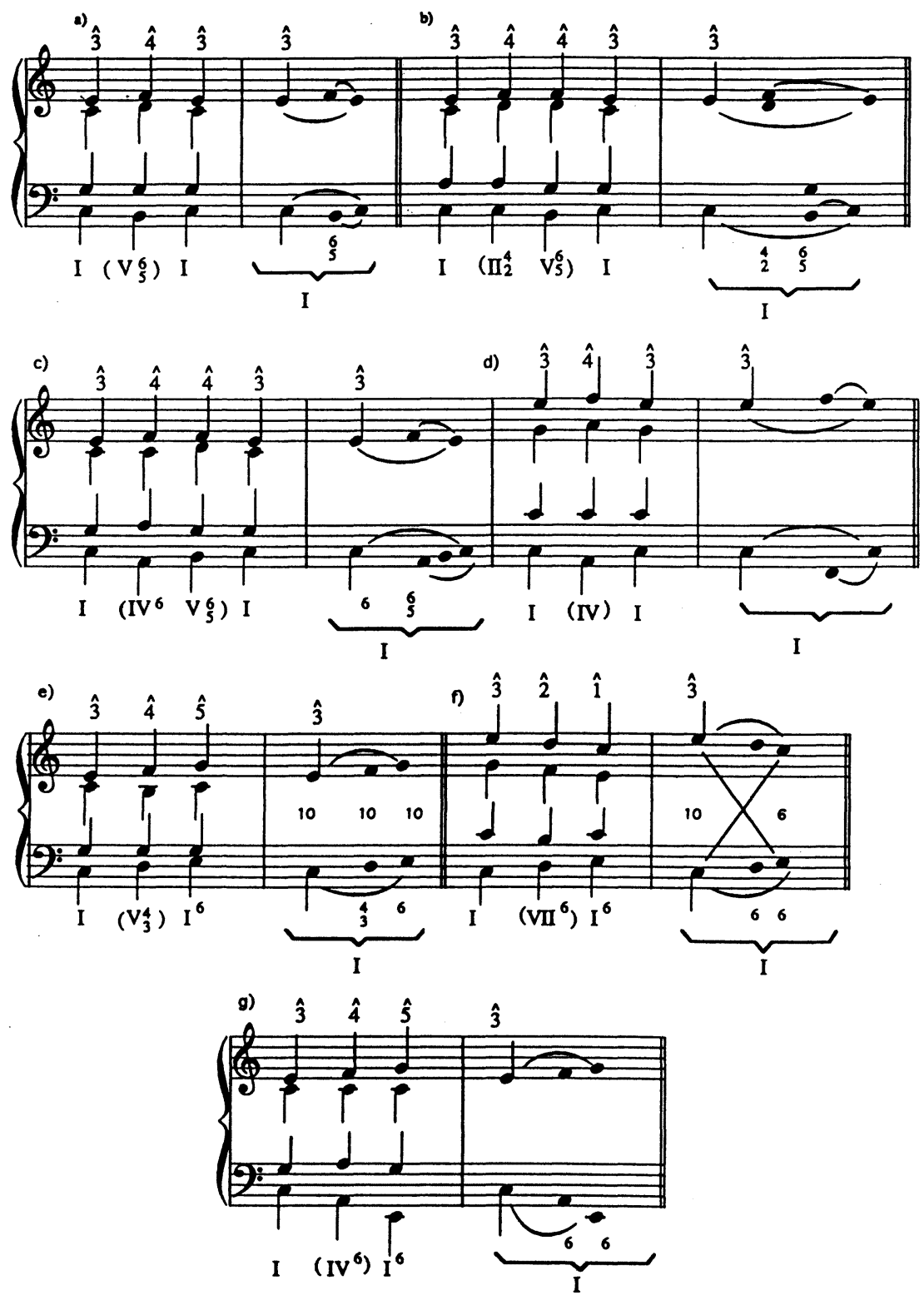

Exemple 7: Tonique. 

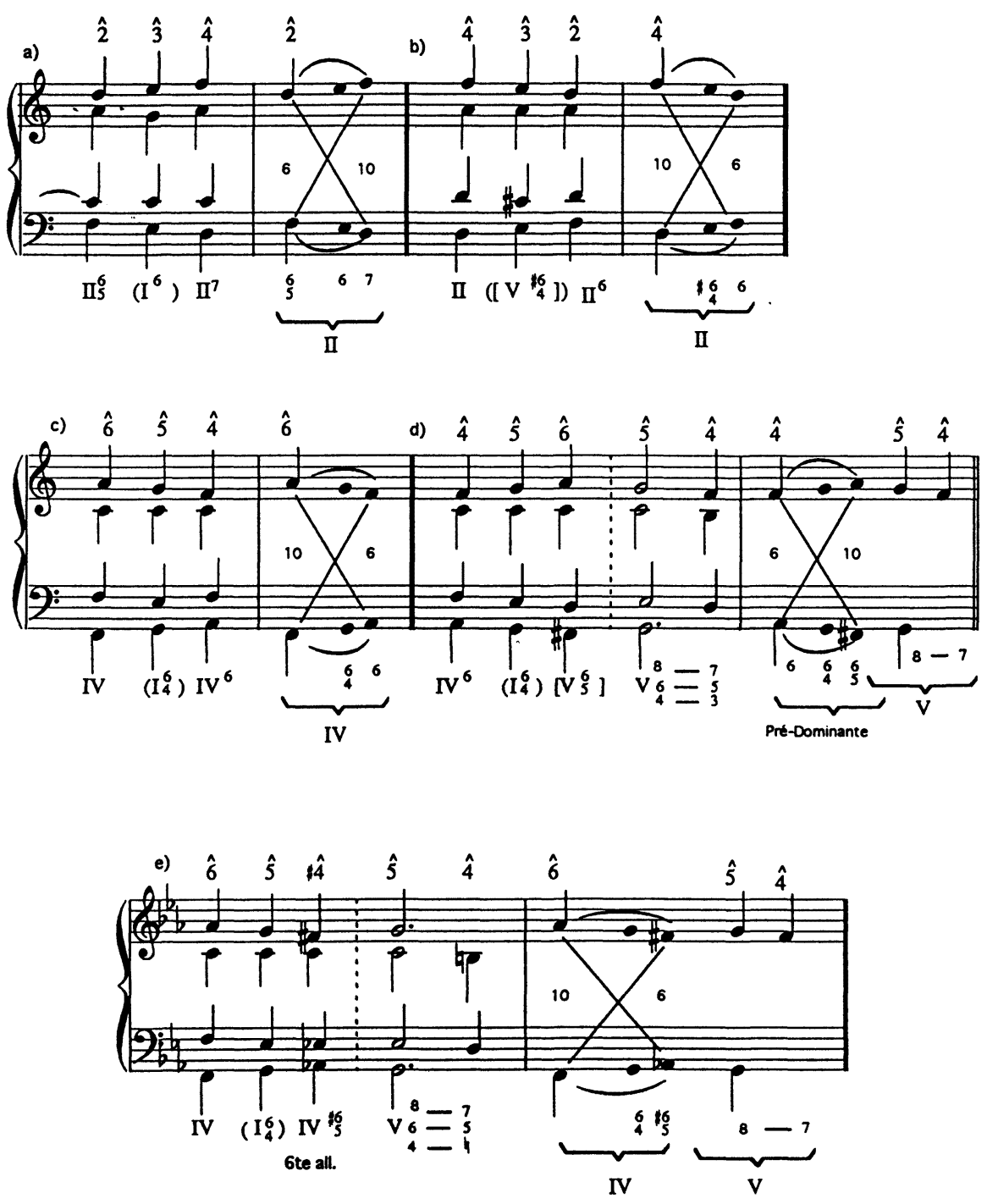

Exemple 8: Pré-dominante. 

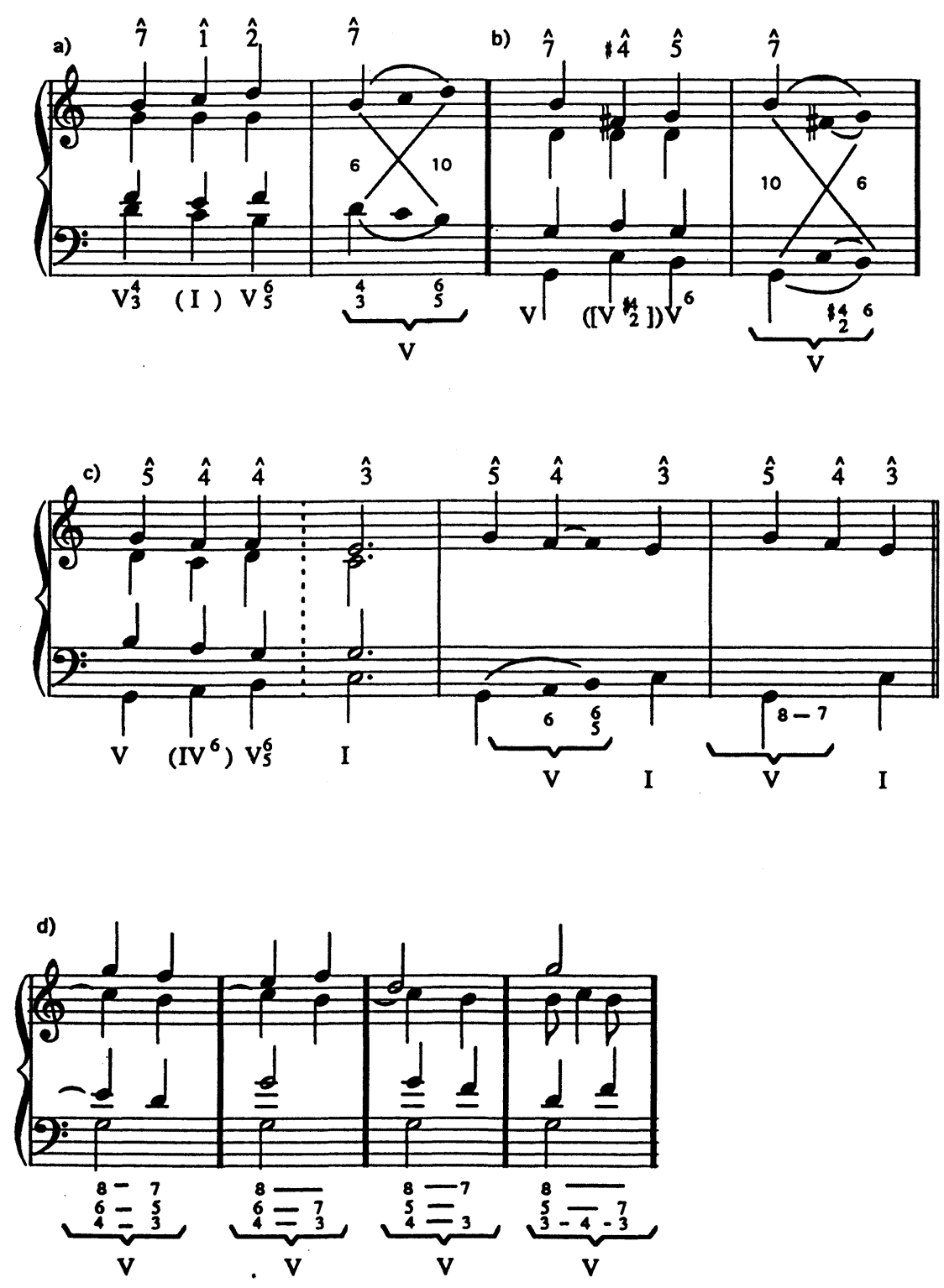

Exemple 9: Dominante. 


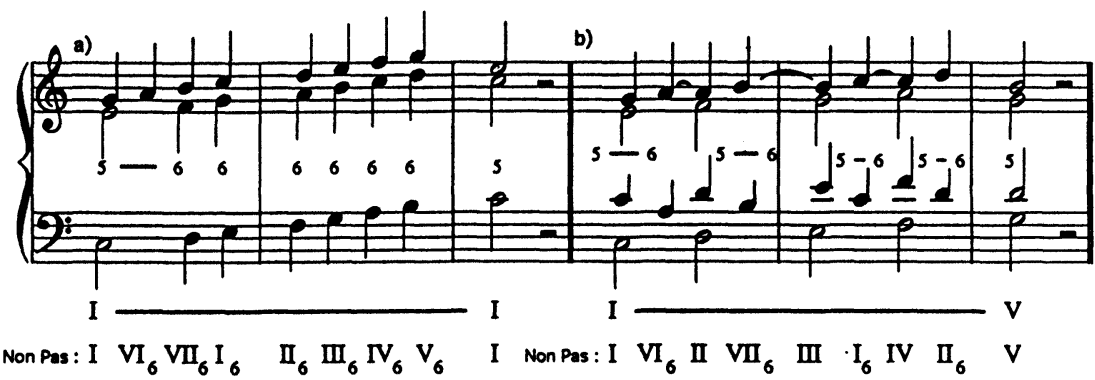

Exemple 10 : Modèles d'intervalles.

La prolongation en force dans les réductions est illustrée au moyen d'une notation graphique inspirée de la notation schenkérienne. Les notes sans hampe représentent les éléments de la progression qui résultent du mouvement contrapuntique et les signes de phrasé expriment la subordination de ces notes à l'accord structurel prolongé. Les notes pourvues d'une hampe identifient la structure prolongée. L'attribution de hampes aux notes de la voix supérieure étant toujours contextuelle, il va se soi que les choix effectués dans le cas des exemples ci-dessous ne sont pas des choix absolus.

Les principes contrapuntiques illustrés par l'exemple 7 appartiennent à la deuxième et à la troisième espèce, respectivement, la note de passage et la broderie (Nebennote) ${ }^{57}$. Aux exemples $7 \mathrm{a}$ et $7 \mathrm{~b}$, la prolongation contrapuntique résulte de la broderie supérieure au soprano et de la broderie inférieure à la basse ; à l'exemple 7c, de la broderie supérieure au soprano et de la broderie disjointe ${ }^{58}$ à la basse ; à l'exemple 7d, de la broderie supérieure au soprano et de la broderie disjointe à la basse (balancement de la quarte); à l'exemple 7e, de la note de

57 Dans la terminologie schenkérienne, la « note voisine » (Nebennote) désigne la broderie. En français, le terme « broderie » implique une broderie complète, c'est-à-dire avec retour au son initial : par exemple, do-broderie ré-retour à do. La broderie incomplète, qui ne revient pas à son point de départ, est généralement désignée en français par le terme « échappée ». Étant donné que la note voisine et la broderie possèdent exactement les mêmes caractéristiques, nous conservons le terme technique français.

58 Nous utilisons le terme «broderie disjointe » pour désigner l'ornementation par mouvement disjoint d'un son parce que ce type de mouvement ornemental est analogue à celui que l'on retrouve dans la broderie. La broderie disjointe étant toujours approchée ou quittée par saut, elle est donc toujours consonante, conformément aux règles du contrepoint d'espèces. En effet, la fonction mélodique de la broderie disjointe est d'offrir un support consonant à la broderie de la voix supérieure. 
passage en mouvement ascendant au soprano et à la basse, d'où les dixièmes parallèles ; à l'exemple 7f, de la note de passage en mouvement descendant au soprano, ascendant à la basse, d'où l'échange de sons entre les voix (10-6) indiqué au moyen des lignes diagonales entrecroisées ; et, finalement, àl'exemple $7 \mathrm{~g}$, de la note de passage en mouvement ascendant au soprano et la technique de la subdivision d'un intervalle large en deux sauts consonants, à la basse.

Aux exemples $8 \mathrm{a}$ à $8 \mathrm{e}$, la prolongation contrapuntique résulte de la combinaison de la note de passage en mouvement ascendant à la note de passage en mouvement descendant. Celle-ci articule un échange entre les voix extrêmes, 6-10 ou 10-6 selon le cas, et un échange chromatique aux exemples $8 \mathrm{~d}$ et $8 \mathrm{e}$. Il en va de même aux exemples $9 \mathrm{a}$ et $9 \mathrm{~b}$. L'exemple $9 \mathrm{c}$ présente deux niveaux de réduction ; le deuxième, le mouvement linéaire $8-7$, indique l'origine mélodique de l'intervalle de septième de dominante, soit par note de passage. L'exemple $9 \mathrm{~d}$ illustre quelques-unes des possibilités d'ornementation de l'accord de dominante, dont la sixte et quarte cadentielle traditionnelle issue du double retard : consonant (6-5) et dissonant (4-3). À l'exemple 10a, la prolongation contrapuntique est au service d'un seul accord structurel, soit une succession de sixtes parallèles en mouvement ascendant qui prolonge l'accord de tonique ; à l'exemple 10b, le retard consonant 5-6 relie des accords structurels différents : l'accord de tonique à l'accord de dominante.

Quelques observations sur la nature du degré harmonique s'imposent avant d'entreprendre l'analyse de l'Inventio 12. La prolongation des accords de tonique, de pré-dominante et de dominante, telle qu'illustrée ci-dessus, n'entraîne pas nécessairement l'implication du statut de degré harmonique pour ces accords. Le postulat selon lequel tous les accords peuvent être prolongés ne signifie nullement que tous les accords sont des degrés harmoniques. À propos de l'identification des degrés harmoniques (Stufen), Schenker met à nouveau en évidence l'interdépendance des principes contrapuntique et harmonique, tel que le démontre le commentaire suivant :

À travers les niveaux de prolongation, c'est parfois l'horizontal qui détermine le cours particulier et la signification du vertical ; et parfois le vertical qui, par sa propre conduite des voix, impose l'horizontal. Ainsi, dans certains cas, nous pouvons parler de l'horizontalisation du vertical et dans d'autres, de la verticalisation de l'horizontal $<\S 79>$.

Il serait donc erroné de lire tous les degrés de la structure de surface sans les différencier, comme si leurs significations et origines étaient identiques. Au contraire, on doit faire la distinction suivante : entre les harmonies qui, d'une façon particulière, s'associent à des diminutions spécifiques près de 
la structure de surface et les harmonies dont les origines expriment des relations fortes dans les niveaux près de la structure fondamentale ${ }^{59}$.

\section{Inventio 12 en la majeur, BWV 783}

La dernière section de cet article sera consacrée à une analyse de l'Inventio 12 en la majeur, BWV 783, à la lumière des observations dégagées plus haut (voir la partition à la fin de cet article). Notre but premier ici étant de mettre en évidence l'importance de la maîtrise des principes contrapuntiques dans la démarche analytique, nous avons choisi de réserver à un article ultérieurl'approfondissement de certaines des potentialités de la théorie schenkérienne, notamment en ce qui concerne la forme ${ }^{60}$ et les motifs ${ }^{61}$. Certaines techniques d'analyse présentées dans le graphe (exemple 17) ne sont évidemment pas accessibles aux étudiants de baccalauréat. Toutefois, tous les choix analytiques présentés ici étant enracinés dans les lois fondamentales du contrepoint rigoureux, nous espérons que l'analyse de l'Inventio 12 convaincra le lecteur de l'importance d'initier les étudiants à ces principes dès le début de leur apprentissage.

La complexité de l'écriture contrapuntique de Bach laisse souvent les étudiants formés à l'école de la verticalité dans la plus complète perplexité. Nous avons précisément choisi l'Inventio 12 parce que le flux linéaire de son écriture

59 « Throughout the prolongation-levels it is sometimes the horizontal which determines the particular course and meaning of the vertical, sometimes the vertical which by its own voiceleading dictates the horizontal. So, in one instance we can speak of the horizontalization of the vertical, in another instance of the verticalization of the horizontal $\langle\S 79\rangle$. / It would be erroneous, therefore, to read all degrees in the foreground without discriminating between them, as though they were all of equal significance and origin. Rather one must make the following distinction : between those harmonies that, in a particular way, serve particular diminutions close to the foreground, and those harmonies which, in their origins, express strong relationships in the levels close to the background. » Schenker, Free Composition, 111-12.

60 Quoique Schenker n'ait que trop peu explicité sa théorie de la forme (voir Free Composition, 128-44), ses écrits sur la fugue et sur la forme sonate sont d'une importance capitale dans son œuvre. Voir « The Organic Aspect of the Fugue », dans Kalib, « Thirteen Essays », 2:245-324, publié à l'origine dans Das Meisterwerk in der Musik II (Munich : Drei Masken Verlag, 1926), 57-95 ; "Organic Stucture in Sonata Form », trad. par Orin Grossman, dans Readings in Schenker Analysis, 38-53, publié à l'origine in Das Meisterwerk in der Musik II (Munich : Drei Masken Verlag, 1926), 45-54. Voir aussi l'excellent article de Janet Schmalfeldt, « Towards a Reconciliation of Schenkerian Concepts with Traditional and Recent Theories of Form », Music Analysis 10, $\mathrm{n}^{\circ} 3$ (1991) : 233-87.

61 Pour une explication du concept de motif chez Schenker, voir, entre autres, Charles Burkhart, «Schenker's "Motivic Parallelisms" ", Journal of Music Theory 22, $\mathbf{n}^{\circ} 2$ (1978) : 145-75; et Carl Schachter, «Motive and Text in Four Schubert Songs », dans Aspects of Schenkerian Theory, 61-76. 
polyphonique à deux voix résiste à l'approche analytique se limitant àl'attribution de chiffres romains. Nous nous attacherons donc à circonscrire et à expliciter le rôle du contrepoint rigoureux dans la différenciation lucide des fonctions contrapuntiques et harmoniques qui régissent la grammaire tonale. Le lecteur trouvera le graphe (exemple 17) et la partition à la fin de l'article. L'identification des registres suit le modèle présenté à l'exemple 11 :

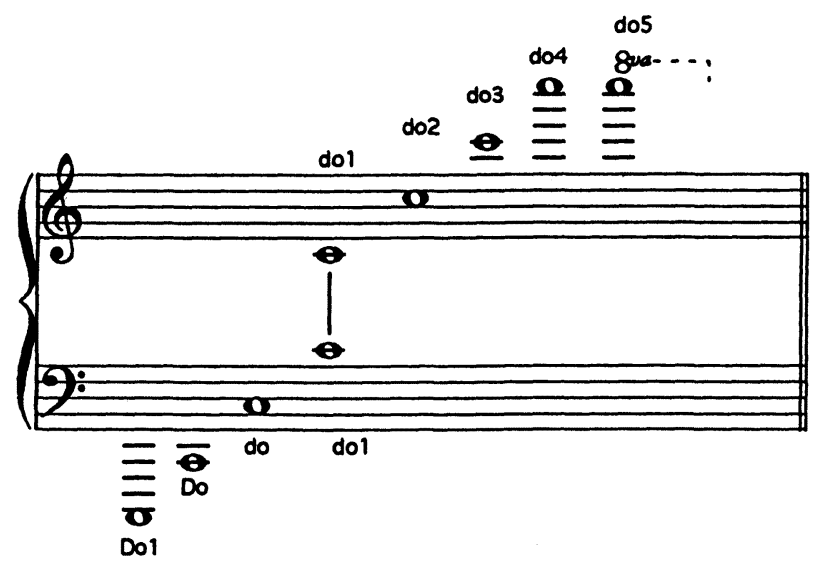

Exemple 11: Identification des registres.

La première démarche analytique consiste à identifier les principales articulations de l'œuvre par l'examen du contour mélodique et par le repérage des points cadentiels. Le thème et l'imitation à la quinte en contrepoint renversable sont présentés aux mesures $1-4$. Un épisode de quatre mesures (mes. 5-8) articule la modulation au ton de fał mineur pour la rentrée du thème et de l'imitation en do mineur (mes. 9-12) ${ }^{62}$. La section centrale (mes. 123-18 ${ }^{1}$ ) présente des diminutions dérivées du thème et de l'épisode des mesures 5-8. La section finale (mes. 18-21) est constituée de la dernière entrée du thème, à la basse, dans la tonalité initiale. Ce survol permet d'identifier le schéma tonal de l'œuvre et de considérer, dès la première étape, les possibilités de prolongation en structure intermédiaire de l'arpège de la basse (Bassbrechung). Cet exercice facilite les prises de position lors des étapes ultérieures de l'analyse. Nous

62 Le terme « modulation » est utilisé ici pour identifier un phénomène ponctuel appartenant à la surface de la musique. La modulation, au sens traditionnel du passage d'une tonalité à une autre, n'existe pas dans la théorie schenkérienne. Voir Carl Schachter, « Analysis by Key : Another Look at Modulation », Music Analysis 6, n 3 (1987) : 289-318. 
proposons la lecture suivante de l'organisation globale de l'œuvre en structure intermédiaire :

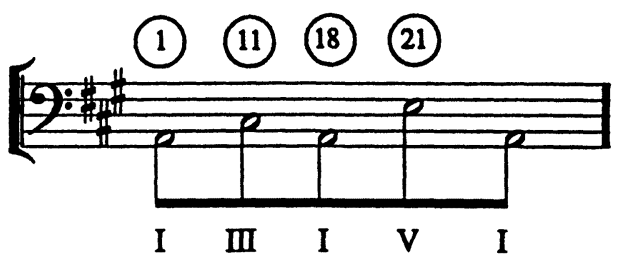

Exemple 12 : Schéma tonal de l'œuvre.

Les exemples 13a à $13 \mathrm{c}$ proposent une lecture des mesures $1-2^{3}$ qui met en évidence la prolongation de l'harmonie de tonique et le mouvement ascendant initial (Anstieg), la ${ }^{1}-s i^{1}-d o \#^{2}$ à la voix supérieure, dont la fonction est d'introduire le son principal (Kopfton), ${ }^{63} d o \sharp^{2}$. Le premier niveau d'interprétation (exemple 13a) identifie le matériel : les harmonies subordonnées VI-II ${ }^{6 / 5-(7)}-V^{7-6-5}$, et les notes ornementales $\mathrm{P}$ : passage, $\mathrm{B}:$ broderie, et $\mathrm{Bi}$ : broderie incomplète. Le Sol\# (mes. $2^{1}$ ) à la basse, est prolongé par l'échange Sol $\$-S i-S o l \#$ et $s i^{1}-s o l{ }^{1}-s i^{1}$, tel que démontré dans le graphe par les lignes diagonales entrecroisées et le contrepoint 10-6-10. Le symbole de croche, associé au ré2 de la mesure $2^{2}$ à la voix supérieure, est le symbole utilisé pour identifier une note voisine ou broderie.

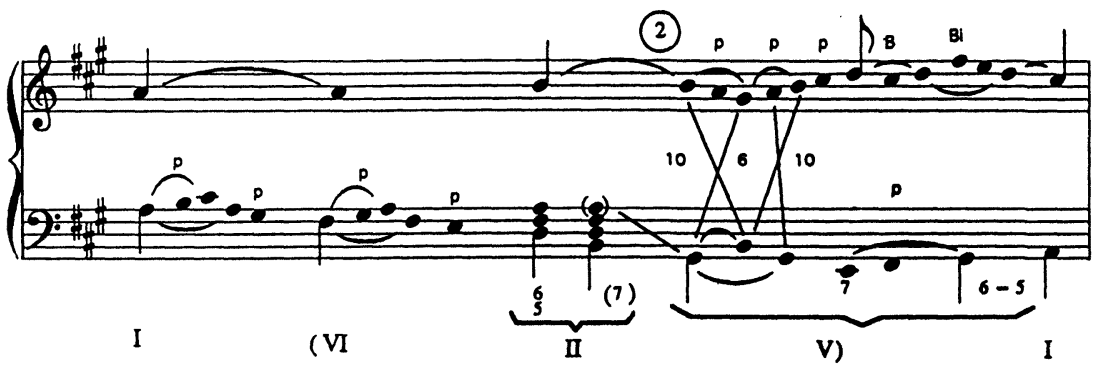

Exemple 13a : Premier niveau de réduction (mouvement ascendant initial et prolongation de l'harmonie de tonique).

63 Dans Free Composition, Ernst Oster traduit Kopfton, qui signifie littéralement « son de tête ", par « primary melodic tone ». Le son de tête est toujours un des degrés de la triade, soit la tierce, la quinte ou l'octave, auquel se greffe la ligne fondamentale (Urlinie) (exemple 1). Comme c'est le cas dans l'Inventio 12 où le son de tête est introduit par un mouvement ascendant initial (Anstieg), il est plutôt rare que le son de tête s'affirme au tout début de l'œuvre. Nous préférons donc « son principal » à « son premier » ou à « son initial ». 
Le graphe de l'exemple 13b clarifie la nature de la prolongation de l'harmonie de tonique, charpente structurelle de ce passage. Le $l a$ (mes. $1^{1}$ ) à la basse est le point de départ du mouvement par tierces descendantes successives, la-fa\#-ré$s i-($ Sol $\$)$, vers $L a$ (mes. $\left.2^{3}\right)$, le but de la progression. Les accords intermédiaires sont des accords-passage qui relient l'accord de tonique à l'accord de septième de dominante en premier renversement, $\mathrm{V}^{6-5}$ (mes. $2^{1-2}$ ). Cet accord est un accord-broderie (Nebennotenharmonie), ici broderie inférieure de la fondamentale de l'harmonie de tonique.

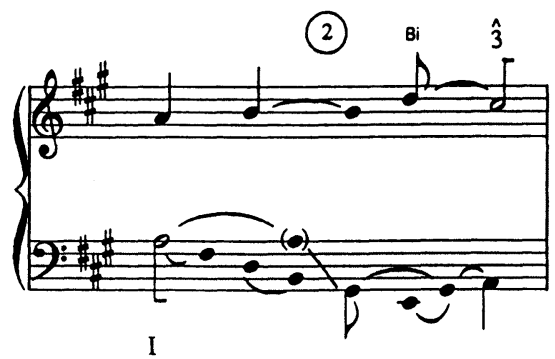

Exemple 13b : Deuxième niveau de réduction (accords-passage et accord-broderie).

L'interprétation proposée à l'exemple 13c clarifie la relation entre structures contrapuntiques et charpente structurelle. La prolongation de l'harmonie de tonique est gouvernée (1) à la voix supérieure par la note de passage $s i^{1}$ dans $l a^{1}$ $s i^{1}-d o \sharp^{2}$, et la broderie incomplète du son principal, $r e^{2}-d o \#^{2}$; et (2) à la basse par la broderie inférieure $l a-S o l \sharp-L a$. La tierce ascendante $l a^{1}-d o \sharp^{2}$, déployée à la voix supérieure, est supportée par le contrepoint $8-10-5^{\circ}-10$. La septième de dominante $r e^{2}$ et la sensible sol\# sont en contrepoint de quinte diminuée et progressent, selon les règles de la conduite des voix, vers la tierce $l a^{1}-d o \#^{2}$ (mes. $2^{3}$ ). Les blanches reliées par le trait diagonal représentent la structure fondamentale.

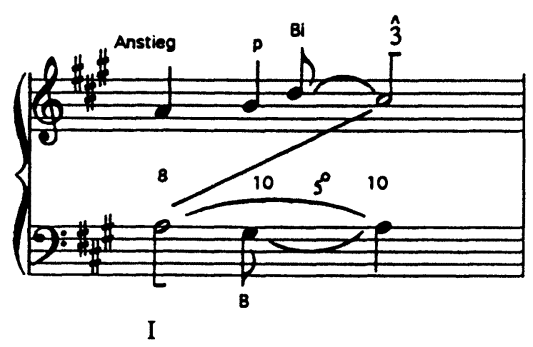

Exemple 13c : Troisième niveau de réduction (relation entre structures contrapuntiques et charpente structurelle). 
Le pont reliant le thème à l'imitation au ton de la dominante (mes. $2^{3-4}$ ) nécessite quelques éclaircissements. Une analyse par attribution de chiffres romains se limiterait à expliquer ce passage par la théorie des accords pivots au service de la modulation au ton de la dominante, soit I- $\left[\mathrm{V}^{7}\right] / \mathrm{V}$ en $l a$ majeur $=\mathrm{IV}-$ $\mathrm{V}^{7}-\mathrm{I}$ en $m i$ majeur (exemple 14a). Cette interprétation ignore la richesse des diminutions utilisées ici par Bach et ne clarifie en rien comment celui-ci a résolu le problème compositionnel de la continuité mélodique entre la présentation du thème (mes. $1-2^{3}$ ) et son imitation à la quinte en contrepoint renversable (mes. $3-4)$.

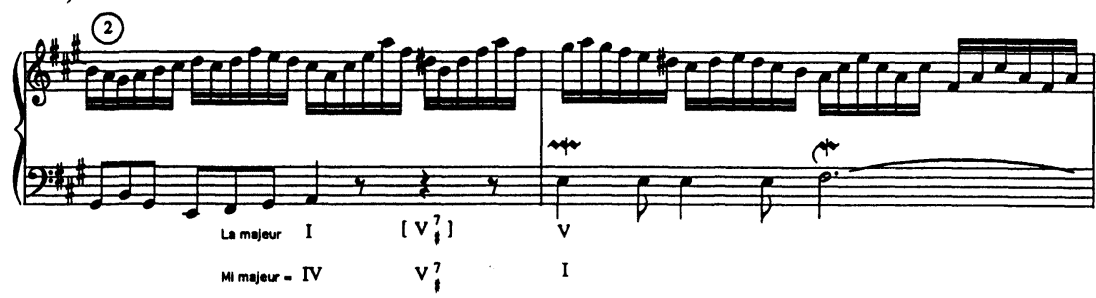

Exemple 14a : Modulation par accords pivots.

L'horizontalisation de l'harmonie de tonique (mes. $2^{3}$ ) introduit $f a \sharp^{2}$, soit la sixième double croche. Certains étudiants iront jusqu'à attribuer le chiffre romain VI à $f a^{2}{ }^{2}$, ou encore $\mathrm{VI}^{6} \mathrm{~s}^{\prime}$ ils conçoivent $l a^{1}$, deuxième double croche de ce groupe de doubles, en tant que voix de basse (exemple 14b). Le graphe de l'exemple $14 \mathrm{c}$ démontre comment le mouvement linéaire 5-6, $m i^{2}-f a \#^{2}$ dans une voix médiane, élimine les quintes parallèles $l a^{1}-m i^{2}$ (mes. $2^{3}$ ) et $s i^{1}-f a \#^{2}$ (mes. $2^{4}$ ). Le lecteur observera que le mouvement linéaire 5-6, deux notes de passage consonantes et adjacentes, est un principe contrapuntique introduit en deuxième espèce.

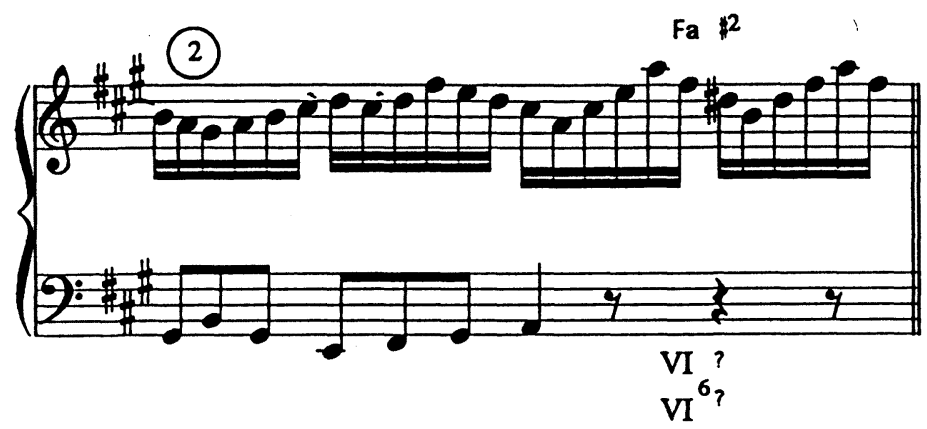

Exemple 14b : Analyse à l'aide de chiffres romains. 


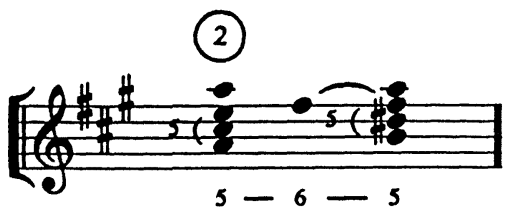

Exemple 14c : Mouvement linéaire 5-6.

Le graphe de l'exemple $14 \mathrm{~d}$ présente la réduction suivante : le transfert de registre de $l a^{1}$ à $l a^{2}$ prépare, par note commune, la septième de l'accord de dominante de la tonalité de mi majeur (mes. $2^{4}$ ) qui se résoudra à sol $\#^{2}$, tierce de la tonique temporaire (mes. $3^{1}$ ). La procédure de déploiement (Ausfaltung) utilisée ici permet d'illustrer les potentialités harmoniques de la voix supérieure. Schenker définit le déploiement en terme d'horizontalisation d'un accord (verticalité) au moyen d'un mouvement reliant un son d'une voix médiane à un son de la voix supérieure suivi du retour à la voix médiane ou vice versa. Le déploiement peut s'appliquer à plusieurs accords en succession ${ }^{64}$. Il est représenté graphiquement comme suit : initialement, la hampe dirigée vers le haut est toujours associée à la note la plus basse de la paire et, inversement, la hampe dirigée vers le bas est associée à la note la plus haute indépendamment de la conduite des voix ; puis, les deux hampes sont reliées par une ligature diagonale. Celle-ci relie ici ré $\#^{2}$ à $l a^{2}$ et $s o l \sharp^{2}$ à $m i^{2}$. Lorsque les hampes sont fixées de la manière prescrite, il est alors possible d'ajouter, soit une hampe supplémentaire dirigée vers le haut ou vers le bas, soit la croche, symbole de la broderie, ou tout autre symbole visant à interpréter la conduite des voix. Dans le graphe de l'exemple $14 \mathrm{e}$, les notes ré $\#^{2}$ et $m i^{2}$ sont pourvues de hampes dirigées le haut mettant en évidence la note de passage ré\# ${ }^{2}$ à l'intérieur du mouvement de tierce ascendant $\left(d o \sharp^{2}-r e ́ \#^{2}-m i^{2}\right)$ qui assure la continuité mélodique entre le thème et son imitation à la dominante dans la voix supérieure.

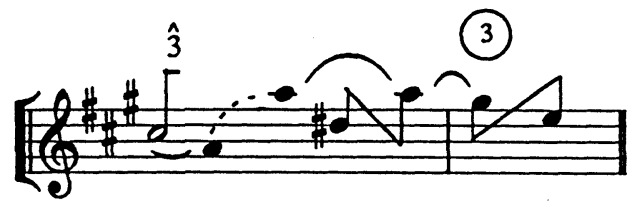

Exemple 14d : Représentation graphique du déploiement. 


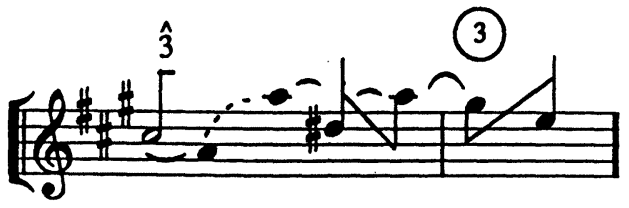

Exemple 14e : Interprétation de la conduite des voix.

La structure bilinéaire qui émerge (première partie de l'exemple 14f) évolue de la sixte $d o \sharp^{2}-l a^{2}$ au triton $r e \#^{2}-l a^{2}$ vers $m i^{2}-s o l \#^{2}$ au premier temps de la mesure 3. Dans la deuxième partie de l'exemple 14f, nous avons transféré la voix supérieure, $l a^{2}-s o l \sharp^{2}$, qui constituent des notes couvrantes (Decktöne), à la voix inférieure, et, ainsi, renversé la progression $6-5^{\circ}-3$, soit $3-4^{x}-6$. Ce renversement clarifie le mouvement de tierce ascendant analysé ci-dessus. Le lecteur observera que ce mouvement mélodique traverse la même distance que le mouvement ascendant initial qui structure le thème, soit l'intervalle de tierce.

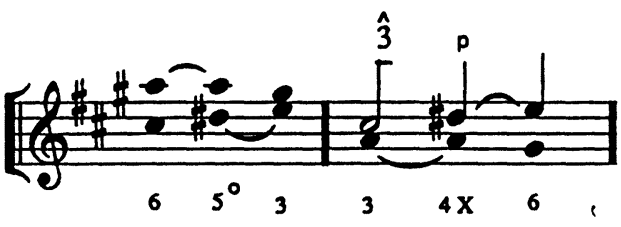

Exemple 14f : Structure bilinéaire et son renversement.
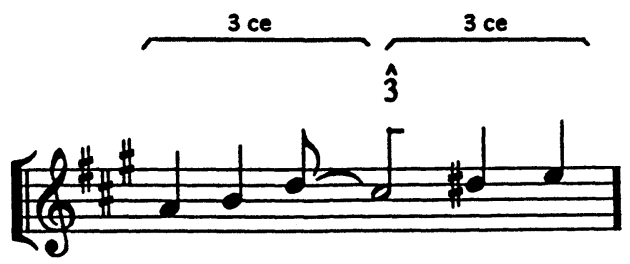

Exemple 14g : Parallélisme entre les mesures $1-2^{3}$ et $2^{3}-3^{1}$.

L'imitation au ton de la dominante (mes. 3-4) intervertit les structures contrapuntiques du thème et de son contrepoint inférieur. Tel que démontré dans le graphe (exemple 17) où l'apparition du thème et de son imitation est indiquée par un crochet à angle droit, nous retrouvons donc $m i^{2}$ à la voix supérieure prolongé par la broderie inférieure $m i^{2}-r e ́ \#^{1}-m i^{2}$ (mes. $3^{1}, 4^{1}$ et $4^{3}$ ), alors que le 
mouvement de tierce ascendant, $M i-F a \#-S o l \#$, prend place au contrepoint inférieur. La note de passage $F a \#$ (mes. $3^{3}-4^{1}$ ) et la broderie incomplète $l a$ (mes. $4^{2}$ ), toutes deux à la basse, constituent le support contrapuntique de ré $\#^{1}$, broderie inférieure de $m i^{2}$ par transfert de registre.

L'épisode des mesures 5-8, de type séquentiel, est divisé en deux sections de deux mesures chacune. La première section (mes. 5-6) présente un groupe de diminutions tirées du thème : la note répétée suivie des arpèges de doubles croches en mouvement descendant à la voix supérieure, et le trait de doubles croches suivi de l'arpège de croches à la voix inférieure. Dans la deuxième section (mes. 7-8), Bach ne conserve que l'arpège de doubles croches en mouvement ascendant qu'il présente en alternance dans les deux voix, ce qui engendre une accélération du rythme des répétitions séquentielles. Les adeptes de la conception verticaliste de l'harmonie interprètent généralement le passage séquentiel en tant que marche harmonique, soit diatonique (non modulante), soit chromatique (modulante), fondée sur la répétition, à un intervalle supérieur ou inférieur, d'une formule mélodique. Cette définition n'éclaire en rien l'essence du fonctionnement de la répétition séquentielle telle qu'elle est élaborée ici et, de manière générale, telle qu'elle se manifeste dans le répertoire tonal.

La séquence consiste en la répétition d'un modèle d'intervalles créé par les voix extrêmes et constitué, par exemple, de l'alternance de consonances (5-6, $5-6 \ldots ; 10-6,10-6 \ldots ;$ etc.), ou d'une série de sixtes ou de dixièmes en mouvement parallèle. Ces paires ou successions d'intervalles sont d'origine purement contrapuntique en ce qu'elles résultent des règles de conduite des voix présentées dans le contrepoint rigoureux. La répétition d'une formule mélodique n'implique pas nécessairement la présence d'un modèle d'intervalles entre les voix extrêmes et, vice versa, la répétition d'un modèle d'intervalles n'est pas toujours associée à une formule mélodique perceptible de manière évidente à la surface de la musique. Il est donc essentiel de différencier la formule mélodique (diminutions, motifs ou fragments caractéristiques) de la conduite des voix qui gouverne la répétition de cette formule. La répétition d'un modèle d'intervalles entre les voix extrêmes prolonge un seul accord structurel ou relie deux accords structurels différents (voir exemples 10a, 10b). Fréquemment utilisé dans le répertoire tonal, ce type de prolongation contrapuntique est toujours au service d'un phénomène appartenant à un niveau plus profond de la structure de l'œuvre.

La prolongation contrapuntique en force dans l'épisode des mesures 5-8 constitue une excellente démonstration du fonctionnement de la répétition d'un modèle d'intervalles. Dans la troisième partie de cet article, nous avons présenté un type particulier de projection compositionnelle: la prolongation contrapuntique au service d'un accord structurel (voir exemples 7 à 9). Dans le cas de l'épisode, la prolongation contrapuntique est au service d'accords structurels : elle relie $\mathrm{V}$ 
à I (mes. 43-7) de même que I à VI (mes. 7-9) ; elle est aussi subordonnée à une structure contrapuntique, plus spécifiquement, le mouvement sous-jacent de dixièmes parallèles. La représentation graphique des mesures $4^{3}-8^{3}$ à l'exemple 15 met en évidence les dixièmes parallèles en mouvement descendant ainsi que les diminutions de la voix de basse, soit les quintes en succession telles qu'elles apparaissent à la faveur du transfert de registre de $r e^{1}, d o \#^{1}$ et $s i$ (mes. $7^{2}, 7^{4}$ et $8^{2}$ ).

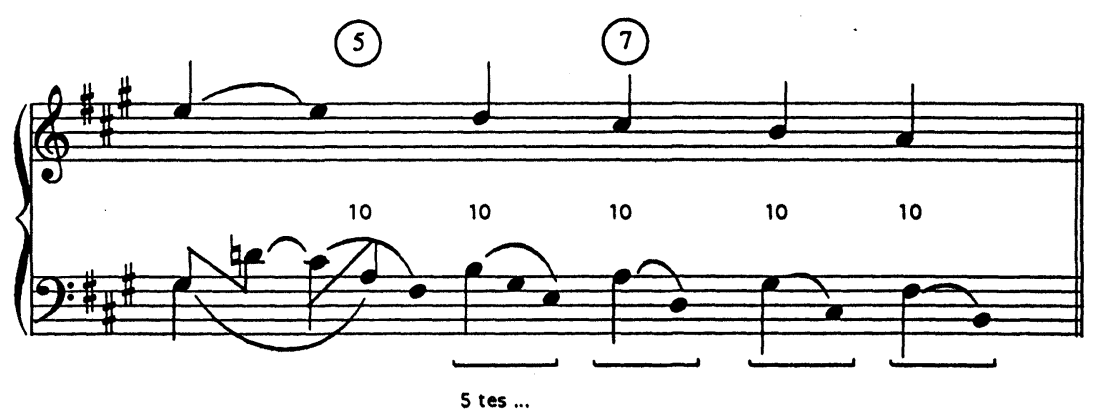

Exemple 15 : Modèle d'intervalles (dixièmes parallèles en mouvement descendant).

La progression sous-jacente, $10-10-10 \ldots$, une progression appartenant au contrepoint de première espèce, constitue la structure contrapuntique sur laquelle se greffe un type de prolongation lui-même enraciné dans les principes de conduite des voix énoncés en contrepoint rigoureux. Plus spécifiquement, le mouvement de la basse par quinte descendante (quarte ascendante) permet d'introduire la dissonance (septième), initialement en tant que consonance, ici une dixième. La structure sous-jacente est alors prolongée au moyen du modèle d'intervalles 10-(5-7) et du modèle d'intervalles 10-(7), respectivement aux mesures 5-6 et 7-8. Ainsi, le principe contrapuntique selon lequel toute dissonance doit être préparée et résolue devient la force motrice de ce passage.

Dans la première moitié de la séquence, le modèle d'intervalles 10-(5-7) prolonge l'accord de septième de dominante de la tonalité initiale, $\mathrm{V}^{6-4 / 2 \ldots 7}$, en structure intermédiaire (mes. $\left.4^{4}-6^{4}\right)$. La résolution à l'harmonie de tonique $\left(\mathrm{V}^{7}-\right.$ $\mathrm{I}$; mes. $6^{4}-7^{1}$ ) rétablit le son principal, $d o \#^{2}$, à la voix supérieure. Le son principal devient le point d'ancrage du deuxième modèle d'intervalles, 10-(7), dont le but ultime est le retour du thème en $f a \sharp$ mineur, degré harmonique VI dans la tonalité initiale de la majeur. La structure intermédiaire $\mathrm{V}^{7}-\mathrm{I}-\mathrm{VI}$ constitue donc la charpente structurelle où se greffent les prolongations contrapuntiques $10-(5-$ 7) et 10-(7) de la structure contrapuntique sous-jacente 10-10-10... Le lecteur observera que la technique du mouvement linéaire 5-6 décrite ci-dessus en 
relation avec un phénomène appartenant à la structure de surface (exemples 14a, $b$ et $c$ ) articule la rentrée du thème en $f a \sharp$ mineur en structure intermédiaire, soit le lien entre I et VI (mes. 7-9), tel que démontré dans le graphe (exemple 16c) ${ }^{65}$.

La deuxième section de l'épisode (mes. 7-8) de même que le retour du thème et de l'imitation au ton de $d o \#$ mineur (mes. 9-11 ${ }^{1}$ ) illustrent un type de prolongation qui exige que nous explorions la structure intermédiaire et la structure fondamentale. En structure de surface, la voix supérieure traverse l'intervalle de quinte en mouvement descendant puis ascendant, $d o \sharp^{2}-f a \sharp^{1}-d o \sharp^{2}$ (mes. $7^{1}, 9^{1}, 11^{1}$ ). Étant donné que le son principal, $d o \sharp^{2}$, reste actif en structure intermédiaire et en structure profonde, ce qui en apparence constitue la voix supérieure est en fait une voix médiane authentique $\left(d o \sharp^{2}-f a \#^{1}-d o \sharp^{1}\right)$. Le son principal $d o \#^{1}$ retient donc sa valeur structurelle pendant toute la durée du passage et gouverne le rapport entre structure fondamentale et structure de surface. Le retour du son principal à la mesure 11 coïncide avec l'arrivée de la tonalité de $d o \sharp$ mineur, dominante du thème en $f a \#$ mineur (mes. 9-11) et degré harmonique III dans la tonalité initiale de l'œuvre. Cette interprétation confirme l'hypothèse émise plus haut (exemple 12), soit la prolongation de l'arpège de la basse (Bassbrechung), I-III-I-V-I, en structure intermédiaire (exemple 16b).

La section centrale (mes. $12^{3}-18^{1}$ ), de type séquentiel, est structurée par le couplage (Koppelung) du son principal $d \sharp^{2}$ à $d o{ }^{1}$. Le couplage, représenté graphiquement par le pointillé en courbe, est le terme utilisé pour désigner un transfert de registre (octave) de longue portée qui implique des éléments de la structure fondamentale ou de la structure intermédiaire. La prolongation en force dans la section centrale est au service du son principal, $d o \sharp^{2}$, du degré harmonique III et du retour à la tonalité de la majeur. Le son principal (mes. $12^{3}$ ) est prolongé par les trois modèles d'intervalles suivants : 6-6(mes. $\left.12^{3}-14^{3}\right), 6-10$ (mes. $14^{3}$ $16^{3}$ ) et $10-10-10$ (mes. $\left.16^{3}-18^{1}\right)$. Les formules mélodiques, ou diminutions, associées aux modèles d'intervalles sont regroupées en trois fragments de deux mesures chacun : les arpèges de doubles croches en mouvement ascendant et descendant (mes. $12^{3}-14^{3}$ ), les diminutions tirées du thème et de son contrepoint inférieur (mes. 143-16 ${ }^{3}$ ) et, finalement, les arpèges de doubles croches en mouvement ascendant (mes. $\left.16^{3}-18^{1}\right)$.

Le modèle 6-6 (mes. $12^{3}-14^{3}$ ) est prolongé par un déploiement d'intervalles (Ausfaltung) à la basse. Les intervalles successifs créent une structure bilinéaire qui évolue selon les règles de la conduite des voix : la sixte (consonance) prépare la quarte augmentée (dissonance) qui se résout à la sixte (consonance). Le deuxième modèle $\left(6-10\right.$; mes. $\left.14^{3}-16^{3}\right)$ est lui aussi prolongé par l'alternance

65 Je tiens à remercier Donald R. McLean (McGill University) pour ses excellentes suggestions sur la conduite des voix de l'Inventio 12 dont plusieurs ont été incorporées dans le graphe. 
consonance-dissonance : $6-\left(5^{\circ}\right), 10-\left(5^{\circ}\right)$; finalement, le modèle $10-10$ est prolongé par la progression par quinte à la basse qui engendre le modèle 10-(7) (mes. 5-8).

Le deuxième modèle d'intervalles constitue le point d'ancrage du retour à la tonalité de la majeur qui s'effectue par la technique du mouvement linéaire 56(mes. 7-9 et $12^{3}-14^{3}$ ) suivi du renversement $6 / 5$ au-dessus du degré harmonique III, respectivement aux mesures $12^{3}, 14^{3}$ et $16^{1}$ (exemple 16c). Le degré harmonique III, actif en structure intermédiaire, est représenté dans le graphe de l'exemple 17 par les parenthèses autour de $d o$ \# et les liaisons en pointillé (mes. $14^{3}$ et $16^{1}$ ). Les quintes $d o \sharp-f a \#$ et $m i-l a$ du deuxième modèle d'intervalles (mes. $14^{3}-15^{1}$ et $15^{3}-16^{1}$ ), représentées dans le graphe par le symbole du déploiement, rappellent la première partie du premier épisode de l'œuvre (mes. 5-6). Il serait sans doute souhaitable d'élaborer davantage sur la relation entre le mouvement de tierce ascendant $d o \#^{1}$, ré $\#^{1}$ et $m i^{1}$ dans une voix médiane (mes. $14^{2}, 15^{1}$ et $15^{3}$ ), représenté dans le graphe par les hampes dirigées vers le haut, et la prolongation de cette même tierce entre la présentation du thème et son imitation à la dominante (mes. $2^{3}, 2^{4}$ et $3^{1}$ ) de même qu'à la rentrée du thème en $d o \sharp$ mineur à la basse (mes. 11-1233). Cependant, étant donné que la présentation de la conception schenkérienne du motif dépasse le but du présent article, nous nous bornerons simplement à souligner le parallélisme motivique qui émerge entre les tierces mentionnées ci-dessus (voir note 61).

Le transfert du son principal $d o^{1}{ }^{1}$ (mes. $\left.18^{1}\right)$ à $d o \#^{2}$ (mes. $18^{2}$ ) articule la dernière rentrée du thème et de son contrepoint, entendus pour la première fois en contrepoint renversé dans la tonalité de $l a$ majeur. La broderie $d o \$^{2}-r e^{2}-d o \#^{2}$ est ainsi entendue à la voix supérieure et le mouvement de tierce ascendant, $\mathrm{La}$ $S i-d o \sharp$, à la voix inférieure (mes. $18^{1}-20^{1}$ ). À la mesure $20^{1}$, le son principal $d o \#^{2}$ est donc supporté en structure de surface par le premier renversement de l'accord de tonique à la basse. Il est ensuite prolongé à la voix supérieure par les mouvements de tierce descendants $d o \#^{2}-s i^{1}-l a^{2}$ ( $l a^{1}$ par transfert de registre) et $m i^{2}-r \hat{e}^{2}-d o \#^{2}$, respectivement aux mesures $20^{1}-21^{1}$ et $21^{1}-21^{3}$. Le premier mouvement de tierce est supporté à la voix inférieure par la broderie supérieure $d o \sharp-r e ́-d o \sharp$ (mes. $\left.20^{1}-22^{1}\right)$. La voix inférieure introduit la broderie incomplète $f a \#-m i, \mathrm{IV}^{6}-\mathrm{V}\left(\right.$ mes. $\left.21^{2}-21^{3}\right)$, où le $f a \#$ est prolongé par le mouvement de tierce descendant fa\#-mi-ré (mes. $\left.21^{2}-21^{3}\right)$. La descente de la ligne fondamentale (Urlinie), qui conclut l'œuvre, est supportée par la cadence finale, V-I, de la structure fondamentale.

Les exemples $16 \mathrm{a}$, b et $\mathrm{c}$ résument l'interaction entre la structure profonde et deux niveaux de structure intermédiaire. Ils illustrent respectivement (1) l'Ursatz de l'œuvre ; (2) un premier niveau de structure intermédiaire où le son principal $d o \#^{2}$ est prolongé par la broderie disjointe I-III-I à la voix inférieure ; et (3) le 
rôle joué par la technique du mouvement linéaire 5-6 en structure intermédiaire dans le premier épisode pour la rentrée du thème en $f a \#$ mineur et dans la section centrale pour le retour à la tonalité initiale de la majeur.

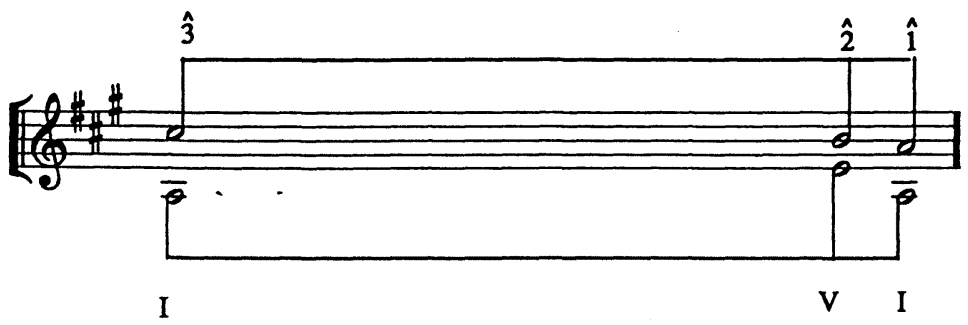

Exemple 16a : Ursatz.

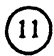

(18)

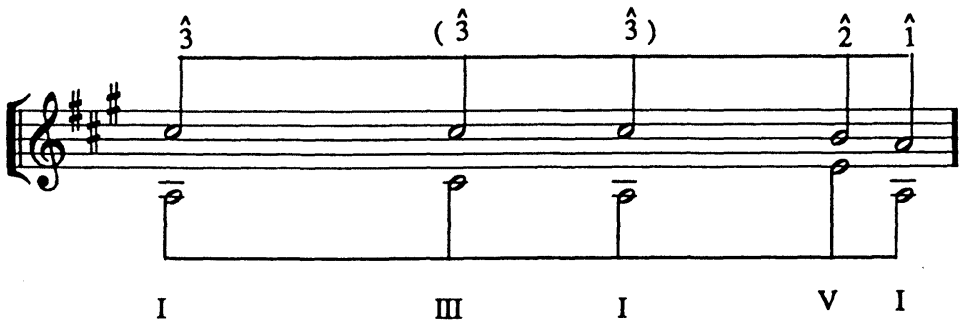

Exemple 16b : Structure intermédiaire I.
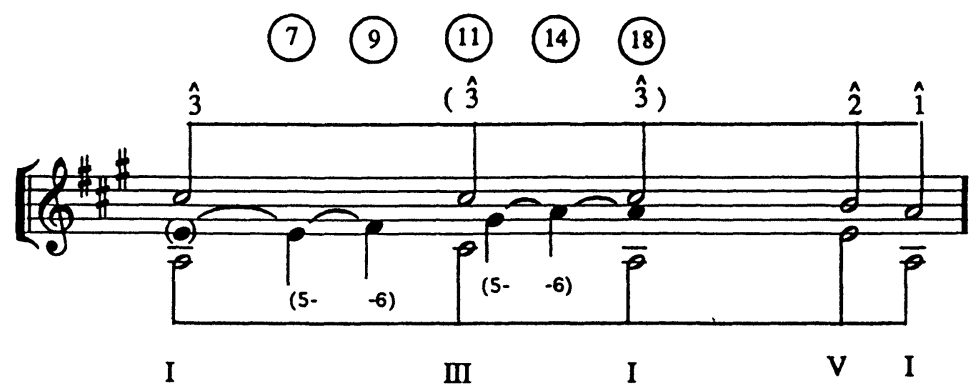

Exemple 16c : Structure intermédiaire II.

Le principe, fondamental en musique tonale, de la préparation consonante de la dissonance et de sa résolution consonante par mouvement conjoint descendant, est introduit, en contrepoint rigoureux, en quatrième espèce. Ce principe, inhérent au fonctionnement de tout processus tonal, assure la motricité de la 

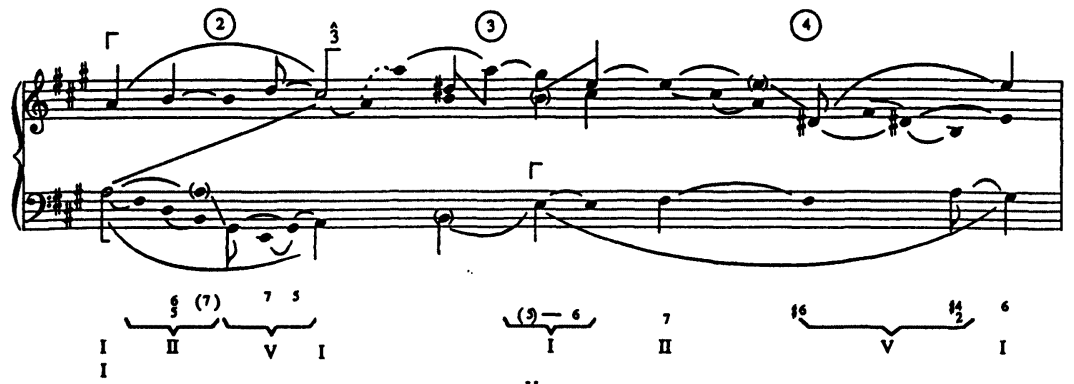

$\underbrace{(9-6)}_{I}$ II

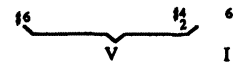

(5)

(6)

(7)

(8)

(9)

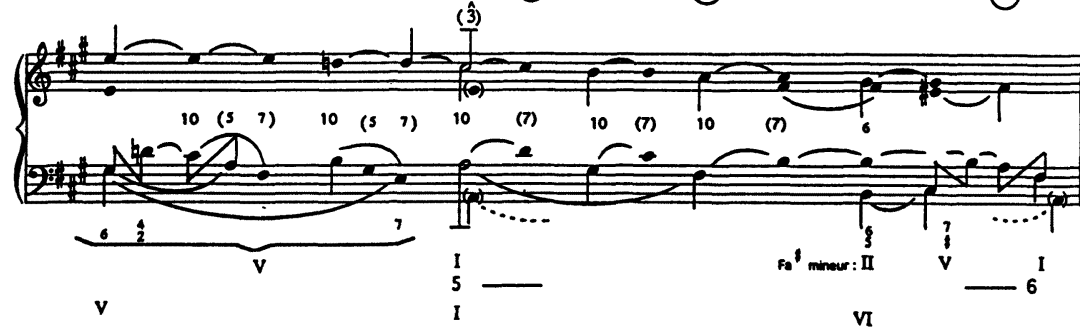

(9) (10)

(11) (3).$\cdots \cdots \cdots$ couplose o o $0^{12}-\infty$

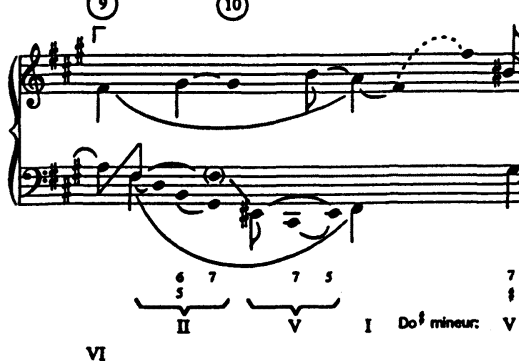

v
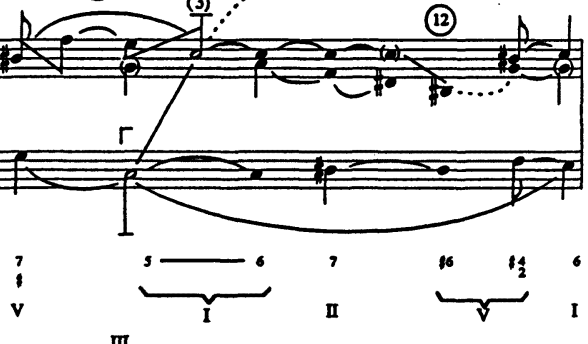

m

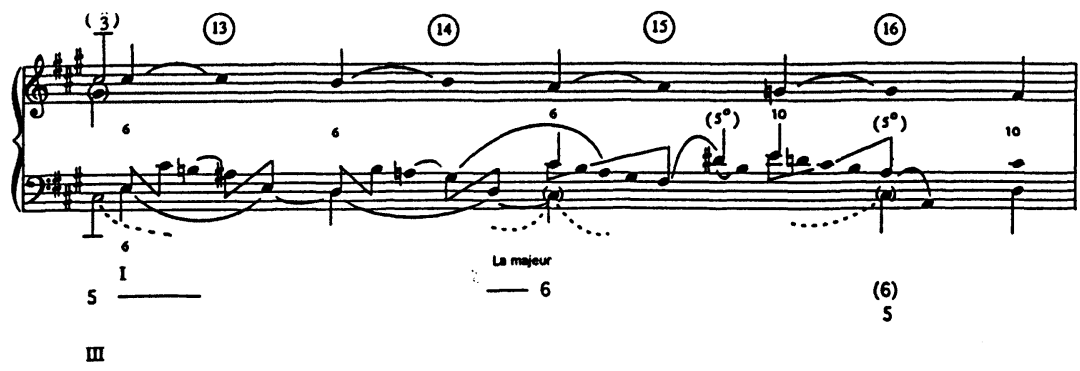



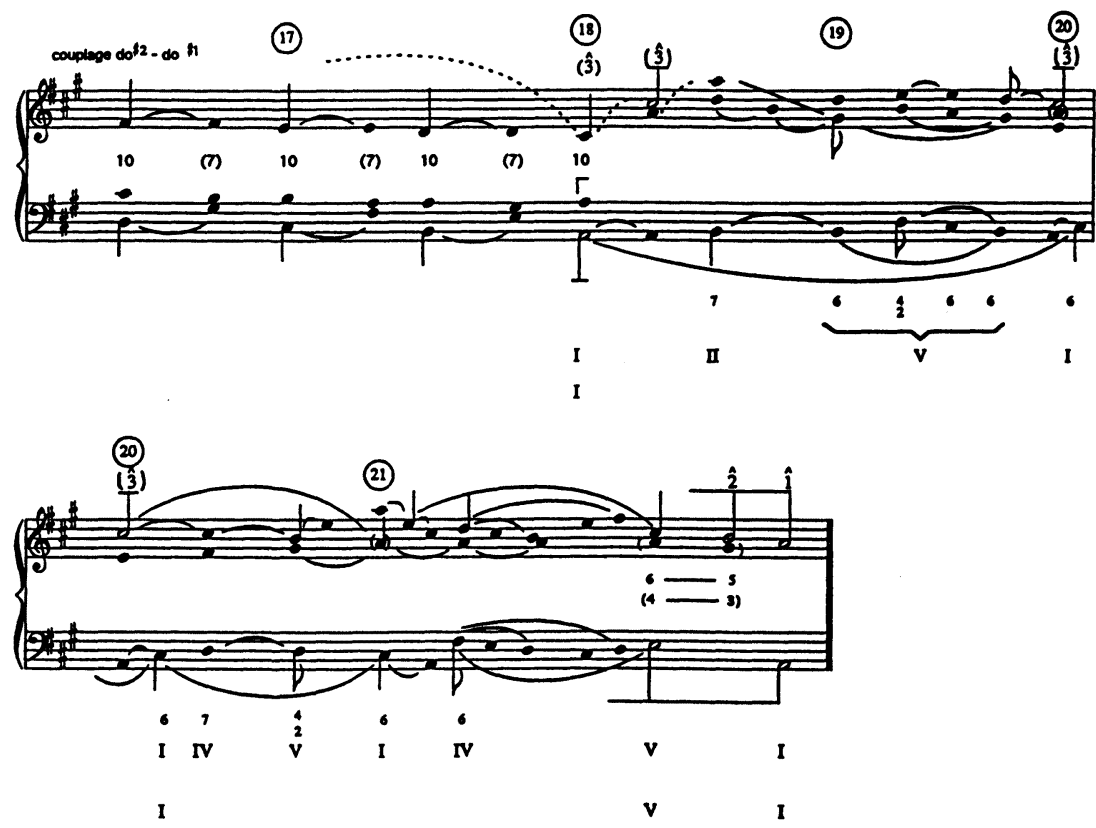

Exemple 17 : Graphe schenkérien.

prolongation contrapuntique en force dans l'épisode et dans la section centrale de l'Inventio 12. Parallèlement, la technique du mouvement linéaire 5-6, qui apparaît dès la deuxième espèce, clarifie les principales articulations de l'œuvre en structure intermédiaire. Nous croyons qu'une analyse de ce matériel par simple attribution de chiffres romains de même qu'une analyse du thème et de son contrepoint sans références aux concepts de note de passage (deuxième espèce) et de note voisine ou broderie (troisième espèce) ne peuvent rendre justice à la complexité contrapuntique et à la fluidité exceptionnelle de cette musique. Nous espérons que la présentation des lois fondamentales du contrepoint rigoureux dans le contexte d'une analyse schenkérienne aura su convaincre le lecteur du bien-fondé de l'étude de cette discipline, et de la pertinence des visées didactiques de Schenker pour l'apprentissage de l'analyse de la musique tonale. 

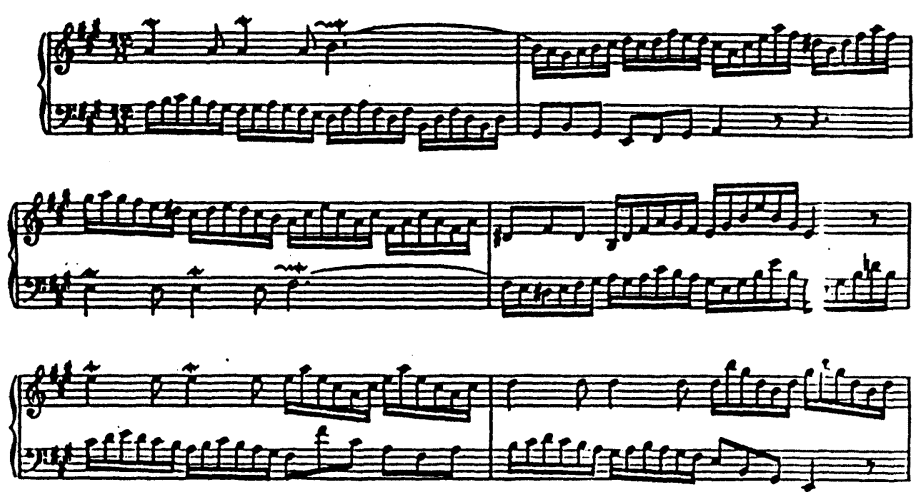

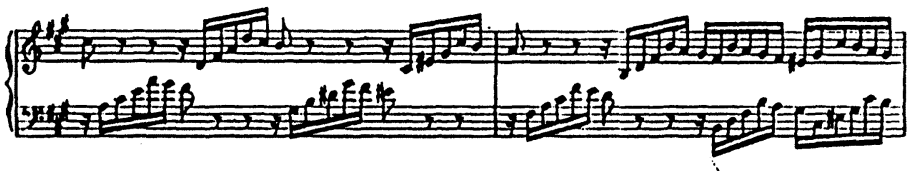

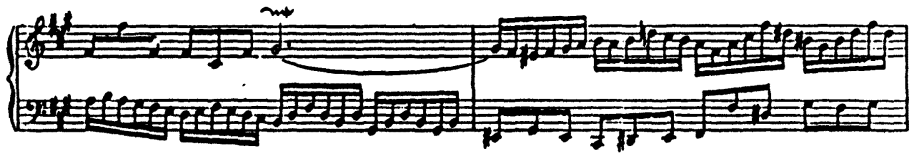

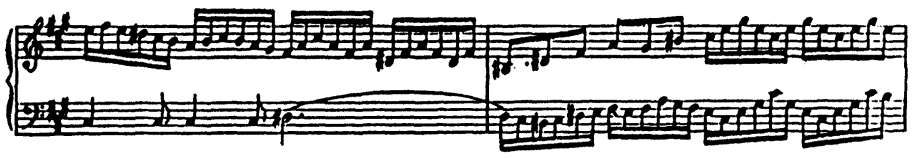

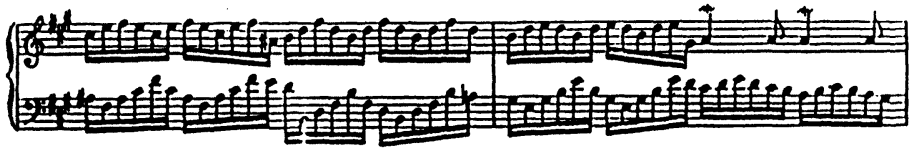

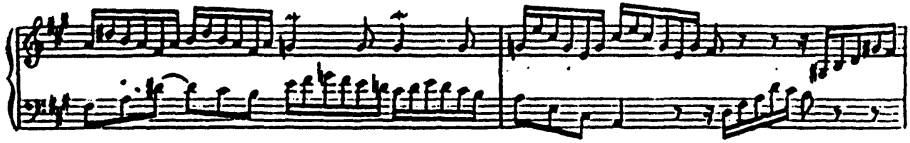
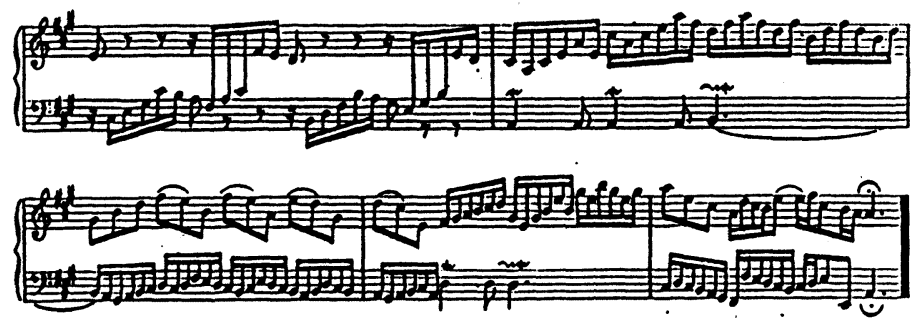

Johann Sebastian Bach, Inventio 12 en la majeur, BWV 783 (Bach-Gesellschaft, vol. 3). 


\section{Résumé}

Nous considérons ici les visées didactiques et certains aspects structurels de la théorie de Heinrich Schenker dans la perspective de l'apprentissage de l'analyse musicale en milieu universitaire de tradition musicologique française. Dans un premier temps, nous posons la question de la pertinence de l'enseignement de l'analyse schenkérienne. Nous mettons en évidence les aspects de la théorie qui la distinguent des théories tonales antérieures et qui représentent des acquis substantiels pour l'étude de la tonalité. Puis, nous évaluons les difficultés inhérentes à la diffusion des idées de Schenker dans les milieux pédagogiques, soit la complexité de son œuvre, l'absence d'un traité d'harmonie à teneur schenkérienne en langue française et l'exploration des niveaux de structure intermédiaire (Mittelgrund). Par la suite, nous définissons les étapes préalables à l'apprentissage des techniques schenkériennes, notamment l'étude des espèces fuxiennes et de l'harmonie, cette dernière enseignée dans une perspective linéaire. Enfin, nous analysons l'Invention 12 en la majeur, BWV 783, de Johann Sebastian Bach. L'analyse met en évidence la relation entre les principes contrapuntiques présentés dans les étapes préalables et les différents niveaux de structure de l'œuvre. Un graphe schenkérien illustre l'interdépendance des niveaux de structure. 